$3-28-2011$

\title{
Detecting Cracked Rotors Using Auxiliary Harmonic Excitation
}

Jerzy T. Sawicki

Cleveland State University, j.sawicki@csuohio.edu

Michael I. Friswell

Swansea University

Zbigniew Kulesza

Bialystok University of Technology, z.kulesza@pb.edu.pl

Adam C. Wroblewski

NASA Glenn Research Center

Follow this and additional works at: https://engagedscholarship.csuohio.edu/enme_facpub

Irhn D. Lekki

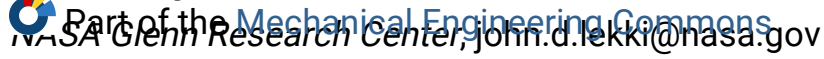

How does access to this work benefit you? Let us know!

\section{Publisher's Statement}

NOTICE: this is the author's version of a work that was accepted for publication in Journal of Sound and Vibration. Changes resulting from the publishing process, such as peer review, editing, corrections, structural formatting, and other quality control mechanisms may not be reflected in this document. Changes may have been made to this work since it was submitted for publication. A definitive version was subsequently published in Journal of Sound and Vibration, 330, 7, (03-28-2011); dx.doi.org/10.1016/j.jsv.2010.10.006

\section{Original Citation}

Jerzy T. Sawicki, Michael I. Friswell, Zbigniew Kulesza, Adam Wroblewski, John D. Lekki. (2011). Detecting cracked rotors using auxiliary harmonic excitation. Journal of Sound and Vibration, 330(7), 1365-1381, doi: dx.doi.org/10.1016/j.jsv.2010.10.006.

This Article is brought to you for free and open access by the Mechanical Engineering Department at EngagedScholarship@CSU. It has been accepted for inclusion in Mechanical Engineering Faculty Publications by an authorized administrator of EngagedScholarship@CSU. For more information, please contact library.es@csuohio.edu. 


\title{
Detecting cracked rotors using auxiliary harmonic excitation
}

\author{
Jerzy T. Sawicki ${ }^{\mathrm{a}, *}$, Michael I. Friswell ${ }^{\mathrm{b}}$, Zbigniew Kulesza $^{\mathrm{c}}$, , Adam Wroblewski $^{\mathrm{d}}$, John D. Lekki ${ }^{\mathrm{e}}$ \\ a Center for Rotating Machinery Dynamics and Control (RoMaDyC), Cleveland State University, Cleveland, OH 44115-2214, USA \\ ${ }^{\mathrm{b}}$ School of Engineering, Swansea University. Swansea SA2 8PP. UK \\ ${ }^{\mathrm{c}}$ Faculty of Mechanical Engineering, Bialystok University of Technology, Bialystok, Poland \\ ${ }^{\circledR}$ ASRC Aerospace Inc., NASA Glenn Research Center, Cleveland, OH 44135, USA \\ 'NASA Glenn Research Center, Cleveland, USA
}

\section{Introduction}

The idea that changes in a rotor's dynamic behaviour could be used for general fault detection and monitoring was first proposed in the 1970 s. Of all machine faults, probably cracks in the rotor pose the greatest danger and research in crack detection has been ongoing for the past 30 years. Current methods examine the response of the machine to unbalance excitation during run-up, run down or during normal operation. In principle, a crack in the rotor will change the dynamic behaviour of the system but in practice it has been found that small or medium size cracks make such a small change to the dynamics of machine system that they are virtually undetectable by this means. Only if the crack grows to a potentially dangerous size it can be readily detected.

Detecting faults in rotating machines presents certain problems that do not occur in fixed structures. First of all, in addition to cracks there are several other types of faults that can be present in a machine, such as a seal rub or a misaligned bearing. Ideally we wish to detect a crack in a machine rotor at an early stage in its development and estimate its size and location. However, compared to static structures, access to a rotor can be limited either in order to contain the working fluid or for safety reasons. When two or more machines are coupled together it is generally sufficient to be able to determine which machine rotor is cracked so that the correct stator is removed to gain access to the rotor. On the other hand, compared to a large static structure, the rotor of a machine is easily excited by the residual out-of-balance that always exists or by the use of active magnetic bearings.

- Corresponding author. Fax: +1216 6879280.

E-mail address: j.sawicki@csuohio.edu (J.T. Sawicki). 
Crack detection methods fall into two groups, pattern recognition and model updating. In the former, a crack model is not directly required, but it is desirable to have some idea of the dynamic behaviour that will result from a cracked rotor in order that it can be recognised in the pattern of behaviour. Historically the 2X response of a machine has been used to detect cracks [1], and if correctly used is an important tool for condition monitoring although care must be exercised as a number of other rotor features also produce a $2 \mathrm{X}$ response. If the model updating approach is applied, then the dynamic behaviour of the rotor is used to update a model of the rotor and in the process determine both the severity and location of any crack. Clearly the crack model used must be adequate for the task. Bachschmid et al. [2] used a least squares method in the frequency domain whereas Markert et al. [3] applied the same approach in the time domain to identify and locate machine faults (including a cracked rotor). A novel technique used in the model updating methods is based on the state estimation. This was used by Kulesza and Sawicki [4] who designed a set of observers based on the FEM model of the rotor augmented with an auxiliary oscillator, to determine the crack presence and location.

There are a number of approaches to the modelling of cracks in beam structures reported in the literature, that fall into three main categories: local stiffness reduction, discrete spring models, and complex models in two or three dimensions. Dimarogonas [5] and Ostachowicz and Krawczuk [6] gave comprehensive surveys of crack modelling approaches. Friswell and Penny [7] considered the performance of various crack models in structural health monitoring. Relating crack size to shaft stiffness is not easy and Papadopoulos and Dimarogonas [8] and Jun et al. [9] have proposed crack models based on fracture mechanics. Papadopoulos and Dimarogonas [8] provided a full $6 \times 6$ flexibility matrix for a transverse surface crack. Mayes and Davies [10] provided a simple relationship between the crack depth and the reduction in stiffness. Keiner and Gadala [11] compared the natural frequencies and orbits of a simple cracked rotor using a beam model and two possible finite element models of the crack. Bachschmid and Pennacchi [12] edited a special issue of MSSP that considered the modelling and analysis of breathing cracks. Although the accuracy of the models of idealised breathing cracks is well established, there are two key difficulties for condition monitoring. First, the stiffness reduction due to a crack is very localised, whereas the operating deflection shapes of a typical machine at its running speed are global. Thus the details of the crack model are less important than the global increase in compliance. Second, real cracks do not propagate in a totally predictable way; the uncertainty in the details of the crack mean that quantitative compliance estimation is difficult.

If the vibration due to any out-of-balance forces acting on a rotor is greater than the static deflection of the rotor due to gravity, then the crack will remain either opened or closed depending on the size and location of the unbalance masses. In the case of the permanently opened crack, the rotor is then asymmetric and this condition can lead to stability problems. If the vibration due to any out-of-balance forces acting on a rotor is less than the static deflection of the rotor due to gravity the crack will open and close (or breathe) as the rotor turns. This is the situation that exists in the case of large horizontal machines and this paper will concentrate on this condition.

Active magnetic bearings (AMB) have been used in high-speed applications or where oil contamination must be prevented, although their low load capacity restricts the scope of applications. Iwatsubo et al. [13] proposed that an external force could be used to detect cracks in the shaft of a machine. If the applied force is periodic, then the presence of the crack generates responses containing frequencies at combinations of the rotor spin speed and applied forcing frequency [14-20]. The excitation by unbalance and AMB forces produces combinational frequencies between critical speeds of the shaft, the rotor spin speed and the frequency of the AMB excitation. The key is to determine the correct excitation frequency to induce a combinational frequency that can be used to identify the magnitude of the time-dependent stiffness arising from the breathing mode of the rotor crack. Although AMBs are often used to excite the shaft, other methods such as piezoelectric actuation applied through auxiliary bearings could be used. Ecker and Tondl [21] used a harmonic variation in support stiffness to increase the speed at which a machine became unstable.

This paper has three important contributions. First, the paper proposes the harmonic balance method as an alternative to the multiple scales analysis used by previous authors to estimate the combination frequencies and associated response amplitudes for a cracked rotor system excited by unbalance and AMBs. Second, the combinations of frequencies that occur in the response are verified on a more complex simulated and experimental system than previously used with more than one resonance frequency. Simulation is used to further investigate the effect of the excitation frequency, measurement noise and modelling errors. Finally, the presented experimental data provide strong evidence for potential application of the described approach for on-line crack rotor detection.

\section{Equations of motion of the cracked rotor}

The analysis of a rotor-bearing system may be performed in fixed or rotating coordinates. If neither the bearings and foundations nor the rotor is axi-symmetric then the resulting differential equations, whether described in fixed or rotating coordinates, will be linear equations with harmonic coefficients. Typically foundations of a large machine will be stiffer vertically than horizontally and in this case the cracked rotor will not be axi-symmetric when the crack is open. Thus there is no compelling reason to used fixed or rotating coordinates for the analysis. To determine the stiffness of the rotor as the crack opens and closes it is easier to work in coordinates that are fixed to the rotor and rotate with it. The reduction in stiffness due to a crack is then calculated in directions perpendicular to and parallel to the crack face, and these directions will rotate with the rotor. Having determined the rotor stiffness in rotating coordinates we transform the stiffness matrix to fixed coordinates and join the stiffness to the system inertia to obtain the equation of motion in fixed coordinates. 
The equations of motion will be developed by assuming that the foundation has no internal degrees of freedom and that the rotor is represented by a shaft-line model. Thus all of the degrees of freedom are located on the rotor. The extension to general systems is straightforward and requires the transformation from rotating to stationary coordinates which is only applied to those degrees of freedom located on the rotor.

Let the stiffness matrix in rotating coordinates for the uncracked rotor be $\tilde{\mathbf{K}}_{0}$ and the reduction in stiffness due to a crack be $\tilde{\mathbf{K}}_{c}(\theta)$, where $\theta$ is the angle between the crack axis and the rotor response at the crack location and determines the extent to which the crack is open. Thus the stiffness of the cracked rotor is

$$
\tilde{\mathbf{K}}_{\mathrm{cr}}=\tilde{\mathbf{K}}_{0}-\tilde{\mathbf{K}}_{c}(\theta) \leftarrow
$$

This stiffness matrix is transformed from rotating to fixed coordinates using the transformation matrix $\mathbf{T}(\Omega t)$, to give, assuming the uncracked rotor is axi-symmetric

$$
\mathbf{K}_{\mathrm{cr}}=\mathbf{T}^{\mathrm{T}} \tilde{\mathbf{K}}_{0} \mathbf{T}-\mathbf{T}^{\mathrm{T}} \tilde{\mathbf{K}}_{c}(\theta) \mathbf{T}=\mathbf{K}_{0}-\mathbf{K}_{c}(\theta, t) \leftarrow
$$

Let the deflection of the system be $\mathbf{q}=\mathbf{q}_{\mathrm{st}}+\mathbf{q}_{\mathrm{dy}}$ where $\mathbf{q}_{\mathrm{st}}$ is the static deflection of the uncracked rotor due to gravity, and $\mathbf{q}_{\text {dy }}$ is the dynamic deflection due to the rotating out of balance and the effects of the crack. Thus, $\dot{\mathbf{q}}=\dot{\mathbf{q}}_{\text {dy }}$ and $\ddot{\mathbf{q}}=\ddot{\mathbf{q}}$ dy , and the equation of motion for the rotor in fixed coordinates is

$$
\mathbf{M} \ddot{\mathbf{q}}_{\mathrm{dy}}+(\mathbf{D}+\mathbf{G}) \dot{\mathbf{q}}_{\mathrm{dy}}+\left(\mathbf{K}_{0}-\mathbf{K}_{c}(\theta, t)\right)\left(\mathbf{q}_{\mathrm{st}}+\mathbf{q}_{\mathrm{dy}}\right)=\mathbf{Q}_{u}(t)+\mathbf{W}
$$

where $\mathbf{Q}_{u}$ and $\mathbf{W}$ are the out of balance forces, and the gravitational force, respectively. Damping and gyroscopic effects have been included as a symmetric positive semi-definite matrix $\mathbf{D}$ and a skew-symmetric matrix $\mathbf{G}$, although they have little direct bearing on the analysis. If there is axi-symmetric damping in the rotor then there will also be a skew-symmetric contribution to the undamaged stiffness matrix, $\mathbf{K}_{0}$. We refer to Eq. (3) as the "full equations".

The steady-state deflection of the rotor varies over each revolution of the rotor since $\mathbf{K}_{c}$ varies. However, the stiffness reduction due to the crack is usually small, and we may make the reasonable assumption that $\left\|\mathbf{K}_{0}\right\| \gg\left\|\mathbf{K}_{c}(\theta, t)\right\|$. With this assumption the steady-state deflection is effectively constant and equal to the static deflection, $\mathbf{q}_{\text {st. }}$. The second approximation commonly used in the analysis of cracked rotors is weight dominance. If the system is weight dominated it means that the static deflection of the rotor is much greater than the response due to the unbalance or rotating asymmetry, that is $\left|\mathbf{q}_{\mathrm{st}}\right| \gg\left|\mathbf{q}_{\mathrm{dy}}\right|$. For example, for a large turbine rotor the static deflection might be of the order of $1 \mathrm{~mm}$ whereas at running speed the amplitude of vibration is typically $50 \mu \mathrm{m}$. Even at a critical speed the allowable level of vibration will only be $250 \mu \mathrm{m}$. In this situation that the crack opening and closing is dependent only on the static deflection and thus $\theta=\Omega t+\theta_{0}$, where $\Omega$ is the rotor speed and $\theta_{0}$ is the initial angle. With these two assumptions, Eq. (3) becomes

$$
\mathbf{M} \ddot{\mathbf{q}}_{\mathrm{dy}}+(\mathbf{D}+\mathbf{G}) \dot{\mathbf{q}}_{\mathrm{dy}}+\left(\mathbf{K}_{0}-\mathbf{K}_{c}(t)\right) \mathbf{q}_{\mathrm{dy}}=\mathbf{Q}_{u}(t) \leftarrow
$$

where $\mathbf{K}_{c}$ is now independent of $\theta$.

\section{Models of breathing cracks}

The breathing crack was initially studied by Gasch [22] who modelled the crack as a hinge. In this model the crack is open for one half and closed for the other half a revolution of the rotor, and the transition from open to closed (and vice-versa) occurs abruptly as the rotor turns. Mayes and Davies [23,24] developed a similar model except that the transition from fully open to fully closed is described by a cosine function, and this model will be used in this paper. Penny and Friswell [25-27] compared the response due to different crack models and considered the effect on the dynamic response of the rotor.

In this paper the fully open crack is modelled by reducing the element stiffness in orthogonal directions (parallel and perpendicular to the crack face). The stiffness matrix of the machine when the crack is open, in rotating coordinates, is then $\tilde{\mathbf{K}}_{1}$. If weight dominance is assumed, then the opening and closing of the crack is periodic at the rotor spin speed. In the Mayes model the time dependent stiffness matrix in rotating coordinates is

$$
\tilde{\mathbf{K}}_{c}(t)=0.5 \times\left(1-\cos \left(\Omega t+\theta_{1}\right)\right)\left[\tilde{\mathbf{K}}_{0}-\tilde{\mathbf{K}}_{1}\right] \quad \leftarrow
$$

where $\theta_{1}$ depends on the crack orientation and the initial angle of the rotor. When $\cos \left(\boldsymbol{\Omega} t+\theta_{1}\right)=1$ the crack is fully closed and $\tilde{\mathbf{K}}_{\mathrm{cr}}(t)=\tilde{\mathbf{K}}_{0}$, the uncracked rotor stiffness, where $\tilde{\mathbf{K}}_{\mathrm{cr}}$ is defined in Eq. (1). Thus the rotor is axi-symmetric when the crack is closed. When $\cos \left(\boldsymbol{\Omega} t+\theta_{1}\right)=-1$ the crack is fully open so that $\tilde{\mathbf{K}}_{\mathrm{cr}}(t)=\tilde{\mathbf{K}}_{1}$. Note that when the crack is open the rotor is asymmetric. In this paper we will choose $\theta_{1}=0$, so that the crack is located at the top of the rotor and is therefore closed at $t=0$.

Converting from rotating to fixed coordinates is performed using the transformation given in Eq. (2). The stiffness matrix in stationary coordinates, $\mathbf{K}_{c}(t)$, is a periodic function of time only and the full nonlinear Eq. (3) becomes a linear parametrically excited equation. Mayes and Davies [10] and Penny and Friswell [25] showed that the model generates a constant term plus $1 \mathrm{X}, 2 \mathrm{X}$ and $3 \mathrm{X}$ rotor angular velocity components in the stiffness matrix. 


\section{Auxiliary excitation of the shaft and multiple scales analysis}

Suppose the shaft is now excited by an external force, typically implemented using AMBs. The force applied on the rotor by the AMBs must be included in the equations of motion. Thus Eq. (4) becomes

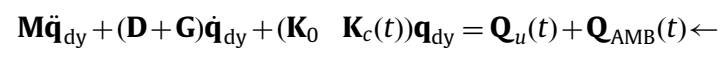

where $\mathbf{Q}_{\text {AмB }}$ is the external forces applied to the rotor by the active magnetic bearing. This force will probably be chosen to be harmonic, either in one or two directions. Other waveforms would be possible if they were perceived to offer some advantage.

The key aspect of the analysis is that the system has three different frequencies, namely the natural frequency (or critical speed), the rotor spin speed and the forcing frequency from the AMB. The parametric terms in the equations of motion (or nonlinear terms in the full equations) cause combinational frequencies in the response of the machine. Mani et al. [16] used a multiple scales analysis to determine the conditions required for a combinational resonance. The analysis is based on the assumptions that the damping, the stiffness variation due to the crack, the nonlinearities and the unbalance force are all small. The first order response is then the sum of two sinusoids consisting of the critical frequency and the AMB excitation frequency, with slowly varying amplitude and phase. In this case a combinational resonance occurs when

$$
\boldsymbol{\Omega}_{2}=\hbar n \boldsymbol{\Omega}_{i} \mid,(\text { for } n= \pm 1, \pm 2, \pm 3, \ldots
$$

where $\Omega$ is the rotor spin speed, $\Omega_{2}$ is the frequendv of the AMB force, and $\omega_{i}$ is a critical frequency of the system. This analysis was based on a two degree of freedom Jeffcott rotor model with weight dominance. Mani et al. [15,16] also considered the effect of detuning, that is when the excitation is close to this exact excitation frequency for resonance, and investigated the effect on the magnitude of the primary resonance close to the critical frequency of the machine. In the examples the running speed of the machine was five times higher than the critical frequency. This ratio is not practical since there is likely to be a second un-modelled resonance below the running speed. Indeed the fact that higher resonances are not modelled is a serious omission, particularly as the combinational resonances are likely to excite any higher frequency resonances.

The multiple scales approach provides excellent results and allows detailed investigation of the system dynamics, for example the effects of detuning from the exact resonance condition. However, multiple scales is not very efficient in the estimation of the steady-state response amplitudes at the combination frequencies for systems with many degrees of freedom. There are also AMB excitation frequencies that do not satisfy Eq. (7) but do produce responses containing combinations of the rotor spin speed and the AMB excitation frequency; this will be demonstrated in the presented case study. Note that these frequencies would be identified by a higher order multiple scales analysis. These difficulties motivate the search for an alternative analysis based on harmonic balance.

\section{Harmonic balance analysis}

The assumptions made for the harmonic balance analysis is that the stiffness variation due to the cracked shaft is periodic with the fundamental frequency corresponding to the rotor spin speed. The unbalance force is synchronous and the AMB force is sinusoidal at a fixed frequency. Thus, in Eq. (6)

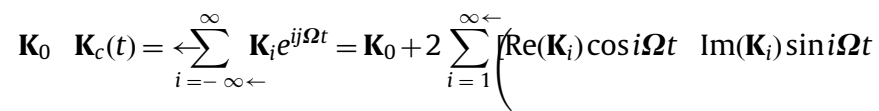

where $\mathbf{K}_{i}=\overline{\mathbf{K}}_{i}$ to ensure that the stiffness matrix is real, the overbar denotes the complex conjugate and $j=\sqrt{1}$. (The exponential form will be preferred for ease of analysis. Note that often the summation in Eq. (8) will be truncated, for exanple in the model of Mayes and Davies [23,24] only the terms up to $|i|=3$ are retained. For an asymmetric rotor, that would model an open crack, only terms up to $|i|=2$ are retained. The unbalance force is

$$
\mathbf{Q}_{u}(t)=\mathbf{Q}_{u 0} e^{j \boldsymbol{\Omega} t}+\overline{\mathbf{Q}}_{u 0} e j \boldsymbol{\Omega} t
$$

where the form of $\mathbf{Q}_{u 0}$ must allow for the synchronous nature of the forcing. The AMB excitation force is assumed to be in a fixed direction in space, and so

$$
\mathbf{Q}_{\mathrm{AMB}}(t)=\mathbf{Q}_{a 0} e^{j \boldsymbol{\Omega}_{2} t}+\overline{\mathbf{Q}}_{a 0} e j \boldsymbol{\Omega}_{2} t
$$

where $\Omega_{2}$ is the excitation frequency and usually only a few elements of $\mathbf{Q}_{a 0}$ are non-zero.

Substituting these expressions for stiffness and force into Eq. (6) implies that the general form of the steady-state response will include combinations of the AMB excitation frequency and the rotor spin speed. Thus,

$$
\mathbf{q}_{\mathrm{dy}}=\leftarrow \sum_{r, s=-}^{\infty} \leftarrow \leftarrow \leftarrow
$$

where the coefficients $\mathbf{q}_{r s}$ are obtained by equating frequencies in the equation of motion

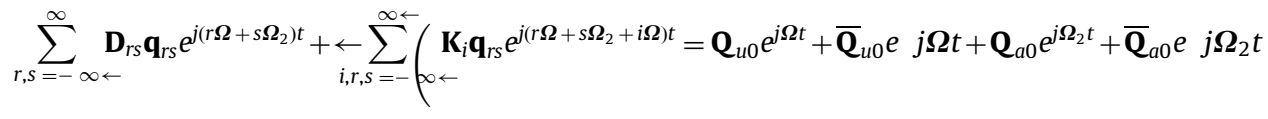


where

$$
\left.\left.\mathbf{D}_{r s}=f\left(r \boldsymbol{\Omega}+s \boldsymbol{\Omega}_{2}\right)^{2} \mathbf{M}+j\left(r \boldsymbol{\Omega}+s \boldsymbol{\Omega}_{2}\right)(\mathbf{D}+\mathbf{G})\right]\right\}
$$

The two summations on the left side of this equation make it difficult to equate frequencies, however a shift $r \rightarrow r \quad i$ in the second summation gives

$$
\sum_{r, s=-\infty \leftarrow}^{\infty} \mathbf{D}_{r s} \mathbf{q}_{r s} e^{j\left(r \boldsymbol{\Omega}+s \boldsymbol{\Omega}_{2}\right) t}+\underset{i, r, s=-\sum_{\infty} \leftarrow \leftarrow}{\infty}\left(\mathbf{K}_{i} \mathbf{q}_{(r \quad i) s} e^{j\left(r \boldsymbol{\Omega}+s \boldsymbol{\Omega}_{2}\right) t}=\mathbf{Q}_{u 0} e^{j \boldsymbol{\Omega} t}+\overline{\mathbf{Q}}_{u 0} e j \boldsymbol{\Omega} t+\mathbf{Q}_{a 0} e^{j \boldsymbol{\boldsymbol { \Omega } _ { 2 } t}}+\overline{\mathbf{Q}}_{a 0} e j \boldsymbol{\boldsymbol { \Omega } _ { 2 } t}\right.
$$

or

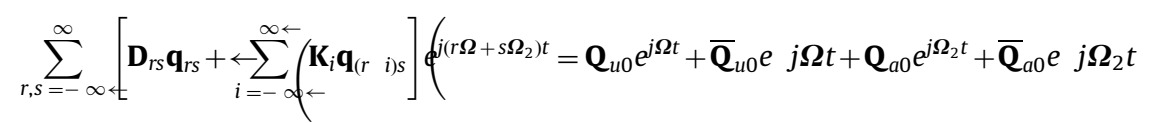

The form of the equations to solve for the unknown vector coefficients, $\mathbf{q}_{r s}$, are now clear. Providing $r \boldsymbol{\Omega}+s \boldsymbol{\Omega}_{2}$ is unique for every combination of $r$ and $s$, the coefficients of each frequency may be equated in Eq. (15). In general the summations for the response and the stiffness variations are infinite. For practical computations these summations in the harmonic balance method are truncated [28,29], and generally this works well because the amplitudes of the coefficients reduce for large $|r|$ or $|s|$. The equations form a pattern that is most easily identified by grouping coefficients of constant $s$ together to give

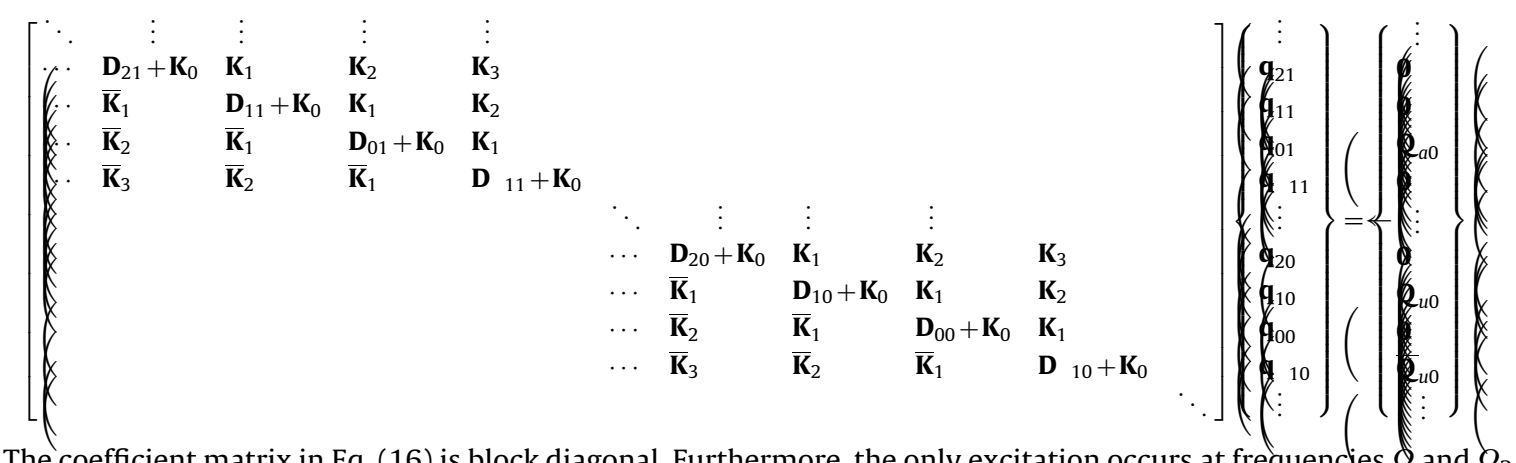

The coefficient matrix in Eq. (16) is block diagonal. Furthermore, the only excitation occurs at frequencies $\Omega_{2}$ and $\Omega_{2}$. This means that $\mathbf{q}_{r s}=\mathbf{0}$ for $|s| \geq 2$.

The $\mathbf{D}_{r s}+\mathbf{K}_{0}$ terms on the diagonal are generally far away from singularity, except for a lightly damped system when $\left(r \Omega+s \Omega_{2}\right)$ is close to a critical frequency of the system, that is

$$
\omega_{i}=r \boldsymbol{\Omega}+s \boldsymbol{\Omega}_{2} \text {, for some } i, r \text { and } s
$$

where $\omega_{i}$ is the $i$ th critical frequency. Hence this condition must be satisfied to obtain a high steady-state response, and is the condition required to set the excitation frequency. This equation is equivalent to that given in Eq. (7) for $s= \pm 1$, and hence the excitation frequencies determined by the multiple scales and the harmonic balance analysis are consistent.

\section{The time simulation}

The analysis thus far has indicated that combinational frequencies are likely to occur in a machine with a breathing crack, excited by a magnetic actuator. Most of the analysis in the literature has been performed on simple two degree of freedom models of the machine, with simplifying assumptions concerning the crack model, gyroscopic effects, higher modes and so on. In order to check the robustness of the frequency content of the machine response a time simulation will be performed on a detailed model of the machine. This will allow realistic features of the real machine to be easily incorporated. To ensure the transient response decays within a reasonable time, damping is added to the bearings and/or disks. The equations of motion are integrated using ode45 in MATLAB. However, the number of degrees of freedom of a detailed finite model is likely to be large, requiring a long computational time to simulate the response. Thus the equations of motion in the rotating frame are reduced using the lower mode shapes of the undamped and undamaged machine, neglecting gyroscopic effects. A sufficient number of modes should be included to simulate the range of excitation frequencies, and also any significant combinational frequencies. This reduction has two beneficial effects; not only are the number of degrees of freedom reduced, leading to a lower computational cost per time step, but also the higher frequencies are removed, thus allowing a larger time step.

The reduction procedure is to calculate the eigenvectors of the undamped and undamaged machine as

$$
\left[-\omega_{0 i}^{2} \mathbf{M}+\mathbf{K}_{0} \boldsymbol{\phi}_{i}=\mathbf{0}\right.
$$

where $\omega_{0 i}$ and $\boldsymbol{\phi}_{i} \pm$ are the $i$ th natural frequency and mode shape. If the lower $r$ modes are retained then the reduction transformation is

$$
\mathbf{T}_{r}=\left\{\boldsymbol{\phi}_{1} \boldsymbol{\phi}_{2} \ldots \boldsymbol{\phi}_{r}\right]
$$


The reduced equations of motion, assuming the mode shapes are mass normalized, are then

$$
\ddot{\mathbf{q}}_{r}+\left(\mathbf{B}_{T}+\mathbf{G}_{r}\right) \dot{\mathbf{q}}_{r}+\left(\mathbf{A}_{0} \quad \mathbf{K}_{r c}(t)\right) \mathbf{q}_{r}=\mathbf{Q}_{r u}(t)+\mathbf{Q}_{\mathrm{rAMB}}(t) \leftarrow
$$

where

$$
\begin{array}{lll}
\mathbf{q}_{\mathrm{dy}}=\mathbf{T}_{r} \mathbf{q}_{r}, & \mathbf{D}_{r}=\mathbf{T}_{r}^{T} \mathbf{D}_{r} \mathbf{T}_{r}, & \mathbf{G}_{r}=\mathbf{T}_{r}^{T} \mathbf{G}_{r} \mathbf{T}_{r}, \quad \mathbf{K}_{r c}=\mathbf{T}_{r}^{T} \mathbf{K}_{c} \mathbf{T}_{r}, \\
& \mathbf{Q}_{r u}=\mathbf{T}_{r}^{T} \mathbf{Q}_{u}, & \mathbf{Q}_{\mathrm{rAMB}}=\mathbf{T}_{r}^{T} \mathbf{Q}_{\mathrm{AMB}},
\end{array}
$$

and $\boldsymbol{\Lambda}_{0}=\operatorname{diag}\left(\omega_{01}^{2}, \omega_{02}^{2}, \ldots, \omega_{0 r}^{2}\right)$ is the diagonal matrix of undamped and undamaged eigenvalues. Note that this reduced equation of motion may also be used in the harmonic balance method described in the previous section.

The equations are integrated until a steady state has been established and then the FFT is calculated. The steady-state response should only contain the excitation and rotor spin frequencies, and the combinational frequencies, and therefore the spectrum of the response should only contain discrete frequencies. However, leakage is likely to occur because of the difficulties in choosing a sample period so that every sinusoidal component in the response has an integer number of cycles in the sample. The effect of leakage may be reduced by using time window functions. Furthermore the sample period must be sufficiently long to ensure that the frequency increment is small enough to distinguish the individual frequency components.

\section{The experimental test rig}

The approach is demonstrated on a test rig utilized at the Center for Rotating Machinery Dynamics and Control (RoMaDyC) at Cleveland State University [19,20]. The photo of rig and its schematic are presented in Fig. 1. The shaft is supported on two ball bearings that are mounted in support housing of conical active magnetic bearings (AMBs). Although the rotor is configured to be supported by AMBs, these are not activated in this study. Each AMB is equipped with two pairs of variable reluctance type position sensors that measure the vibrations in two perpendicular directions. The rig is also equipped with an additional radial AMB that is used as an actuator applying the external force into the rotor. The actuator can be placed at any axial location allowing the external excitation to be applied at almost any point of the shaft. The waveform of this excitation is generated in MATLAB/Simulink environment and transferred to the hardware via dSPACE DS1103 board. As in the supporting AMBs, two pairs of position sensors are mounted on the AMB actuator too. Additional proximity probes can be mounted onto the test rig base, allowing rotor displacement measurements at almost any point along its axis. For the current demonstration

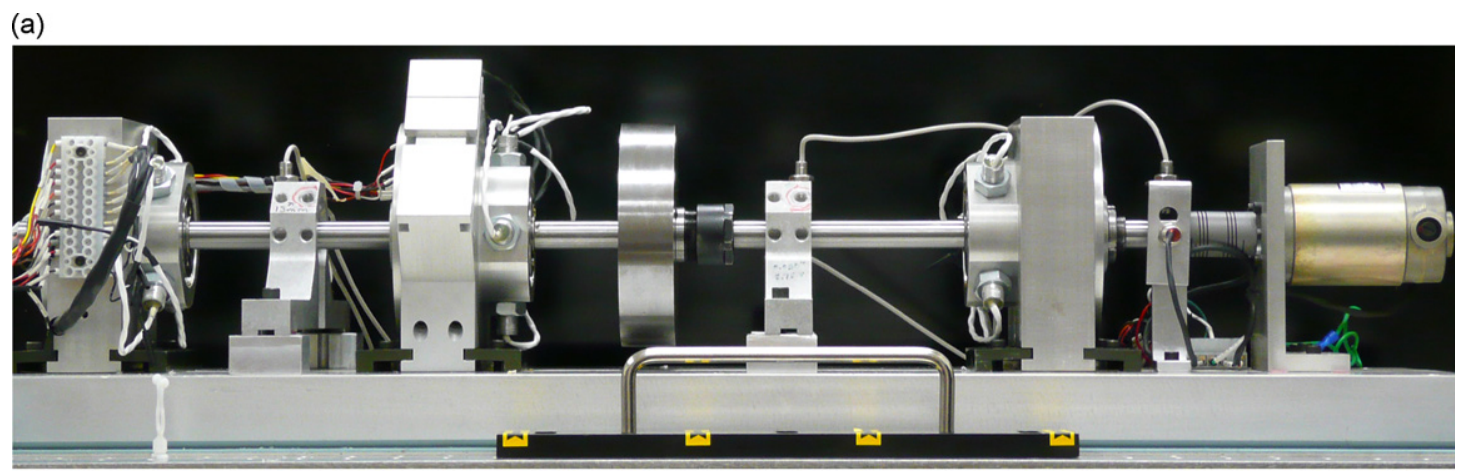

(b)

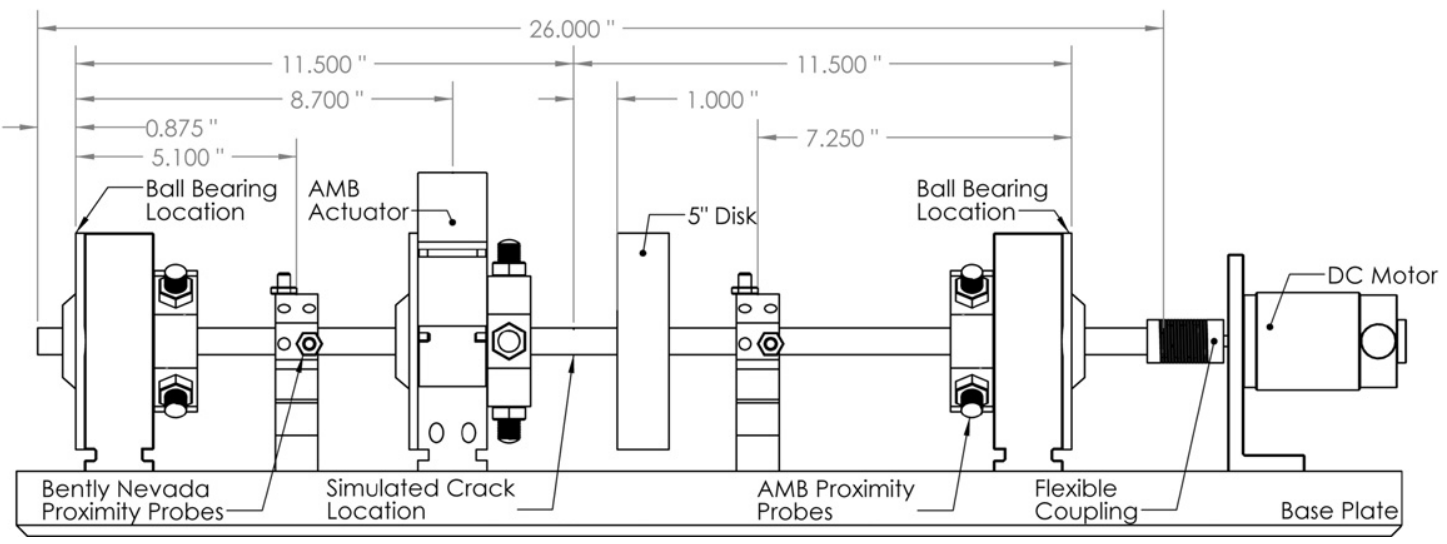

Fig. 1. Photo (top) and CAD drawing of the rotor crack detection rig. 
the actuator is placed close to the crack location. For general applications the actuator must be able to excite the active modes of interest and for particular machines the location should be optimised taking account of any practical limitations that may exist. The 48 V DC, $0-15,000 \mathrm{rev} / \mathrm{min}$ brushed motor controlled by a digital controller is used to drive the rotor. The spin speed and the motor torque are monitored and the controller will shut the power to the motor off in the event of a fault or alarm. The shaft is connected to the motor using a lightweight, flexible coupling that allows radial and axial movement of the shaft.

Two digital controllers provide control loop updates at a $10 \mathrm{kHz}$ frequency and they essentially control and power the magnetic bearings while allowing the user to register useful information about the system under operation. The dSPACE platform is used to have complete control over the experiment by providing access to every sensor signal, user defined control command, and operational parameters.

\section{Simulation and experimental results for the test rig}

The simulated machine is based on the experimental test rig described above. Fig. 2 shows a schematic of the machine and indicates the 30 finite elements used to model the rotor. The shaft diameter is $15.875 \mathrm{~mm}$ and the shaft length is $65.9 \mathrm{~mm}$. The disk has a diameter of $127 \mathrm{~mm}$ and a thickness of $12.7 \mathrm{~mm}$. The diameter of the active magnetic bearing rotors and radial actuator is $47.90 \mathrm{~mm}$. The rotor is made of steel with modulus of elasticity $E=206.8 \mathrm{GPa}$, Poisson's ratio $v=0.3$, and density $\rho=7888.7 \mathrm{~kg} / \mathrm{m}^{3}$. The shaft is running on single row deep groove ball bearings which are modelled as constant stiffness bearings with stiffness $30 \mathrm{MN} / \mathrm{m}$ and damping $10 \mathrm{~N} \mathrm{~s} / \mathrm{m}$ at nodes 2 and 29, as shown in Fig. 2. To approximate the effect of a crack, a notch is cut using technique known as wire electrical discharge machining (EDM) with the wire diameter of $1.14310^{4} \mathrm{~m}$. The notch is located at the shaft between the magnetic actuator and the unbalance disk. The shaft crack is modelled at the 16th element, and its length was taken as $25.4 \mathrm{~mm}$. Two dampers of value $50 \mathrm{~N} / \mathrm{m}$ are added at the location of nodes 12 and 20. These dampers are used to increase damping in the first mode, and hence reduce the time required for the transient response to decay. During the experiments the radial displacements of the rotor are measured in the horizontal and vertical directions. The displacements are taken at the $21 \mathrm{st}$ node. For all simulations the rotor speed was $\Omega=27 \mathrm{~Hz}$ ( $1620 \mathrm{rev} /$ $\mathrm{min})$. For the undamaged rotor, the first two critical bending frequencies are located at $\omega_{1}=36 \mathrm{~Hz}$ and $\omega_{2}=274 \mathrm{~Hz}$. An out of balance with magnitude $10{ }^{3} \mathrm{Nm}$ was added at the disk location (17th node) and, when applied, the active magnetic bearing $(A M B)$ injected a peak harmonic force of $10 \mathrm{~N}$ at the 13 th node. Fig. 3 shows the Campbell diagram for the rotor system and Fig. 4 shows the first four mode shapes at a rotor spin speed of $1620 \mathrm{rev} / \mathrm{min}$.

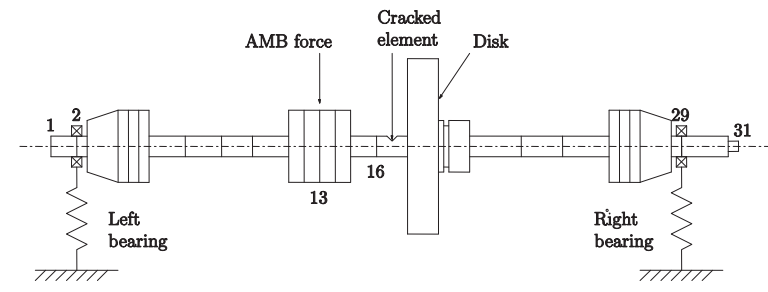

Fig. 2. Finite element model of the rotor system.

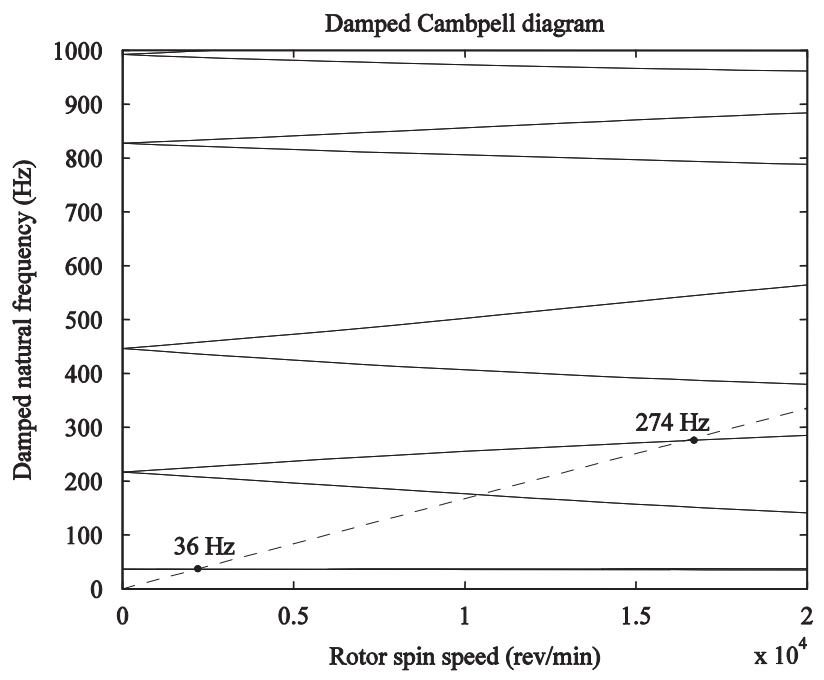

Fig. 3. The Campbell diagram for the rotor system. 


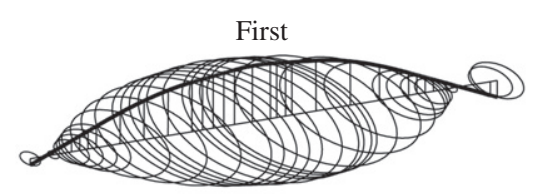

Third

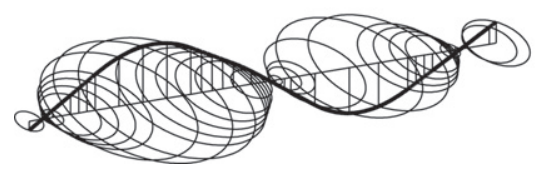

Second

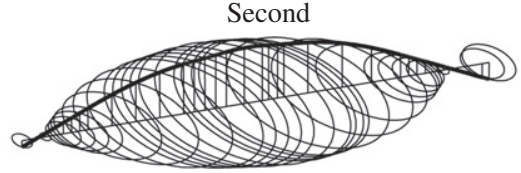

Fourth

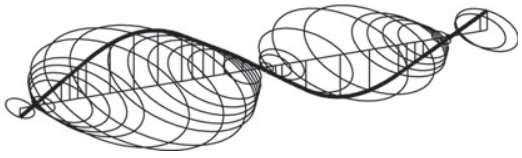

Fig. 4. The first four mode shapes for the rotor system at $1620 \mathrm{rev} / \mathrm{min}$.

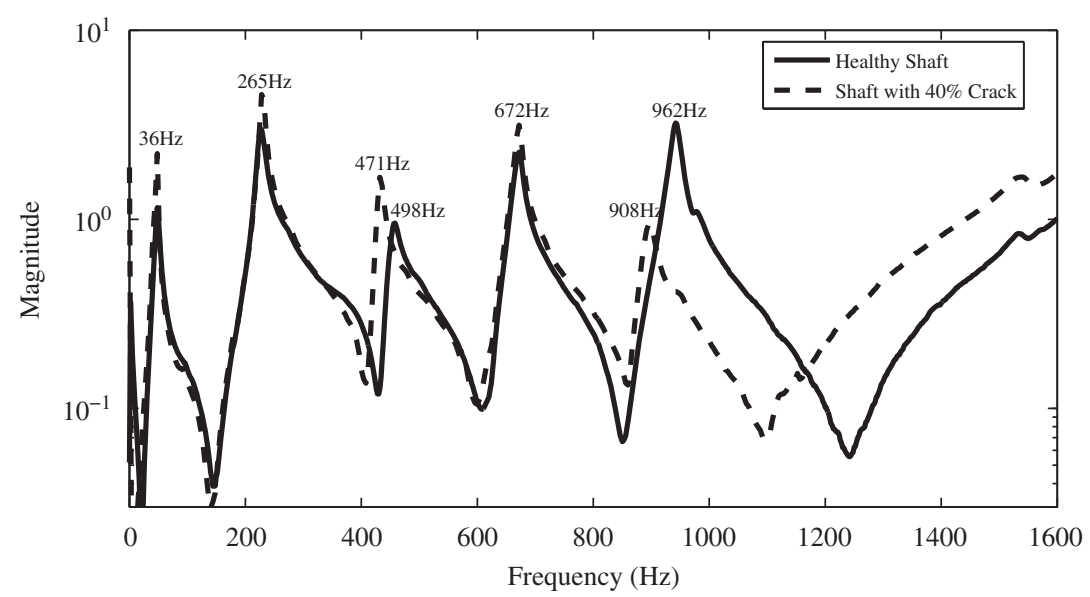

Fig. 5. Measured FFT comparison of the healthy vs. the shaft with a $40 \%$ crack.

Fig. 5 illustrates a comparison of two transfer functions of a non-rotating rotor. These were experimentally acquired through the use of the dynamic signal analyzer with the instrumented hammer and accelerometer. Shown with the solid and dotted lines are the amplitudes of the transfer function for the rotor with no crack and for the rotor with a $40 \%$ crack, respectively. Modes one, two, and four for both uncracked and damaged shafts are in the same locations on the frequency axis. However, the key features of this comparison are the locations of modes three and five. The damaged shaft shows a $27 \mathrm{~Hz}$ shift to the left at the third mode and a $54 \mathrm{~Hz}$ shift to the left at the fifth mode. The amplitudes of the shaft with $40 \%$ crack are generally higher than the healthy shaft's ones which can be expected due to the slight reduction of shaft stiffness.

According to Eq. (11) the response $\mathbf{q}_{\mathrm{dy}}$ of the rotor will include combinations $\omega_{c}$ of the AMB excitation frequency $\Omega_{2}$ and the rotor spin speed $\Omega$, i.e.

$$
\omega_{c}=r \boldsymbol{\Omega}+s \boldsymbol{\Omega}_{2}
$$

As we can see, depending on the excitation frequency and for the given rotor spin speed, the frequency spectra of the rotor vibration response will include different combinational frequencies. However, Eq. (17) suggests, that the amplitude of the response will be maximum when $\left(r \Omega+s \Omega_{2}\right)$ is close to a critical frequency $\omega_{i}$. This restricts the choice of possible excitation frequencies to a limited number only. Fig. 6 shows the candidate external excitation frequencies $\Omega_{2}$ calculated using Eq. (17) for the first two critical frequencies $\omega_{1}=36 \mathrm{~Hz}$ and $\omega_{2}=274 \mathrm{~Hz}$, and for $s= \pm 1, r=0,1, \ldots, 5,6$. To investigate this further and to compare the results obtained by the time simulation and from the harmonic balance, we will consider some examples at different external excitations.

Fig. 7 presents the response of the uncracked rotor with no excitation. The line shows the response obtained by the time simulation, where the model has been reduced to the first 12 modes. The integration is performed over 670 cycles of the rotor spin frequency, and the first 400 cycles are discarded to allow the transients to decay. Note that the number of cycles (270) has been chosen so that there is no leakage effects at the significant frequency at which the response is non-zero. The crosses denote the results from the harmonic balance method and results agree closely with the time simulation. The only response is at the rotor spin speed $\Omega=27 \mathrm{~Hz}$ (marked as 1X). Of course the advantage of using harmonic balance is that the computation is much less.

Fig. 8 shows the response of the undamaged rotor when the AMB excitation force is present. The frequency of this force is $\Omega_{2}=18 \mathrm{~Hz}$, based on an assumed critical frequency of $\omega_{1}=36 \mathrm{~Hz}$, with $n=2$ in Eq. (7), or $r=2$ and $s=1 \mathrm{in} \mathrm{Eq.} \mathrm{(17).} \mathrm{The} \mathrm{response}$ 


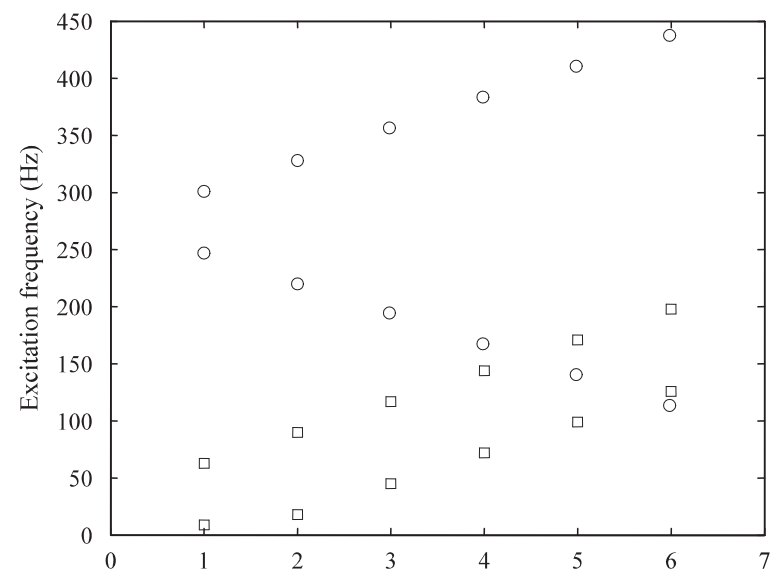

Fig. 6. Candidate external excitation frequencies for mode 1 (squares) and mode 2 (circles).

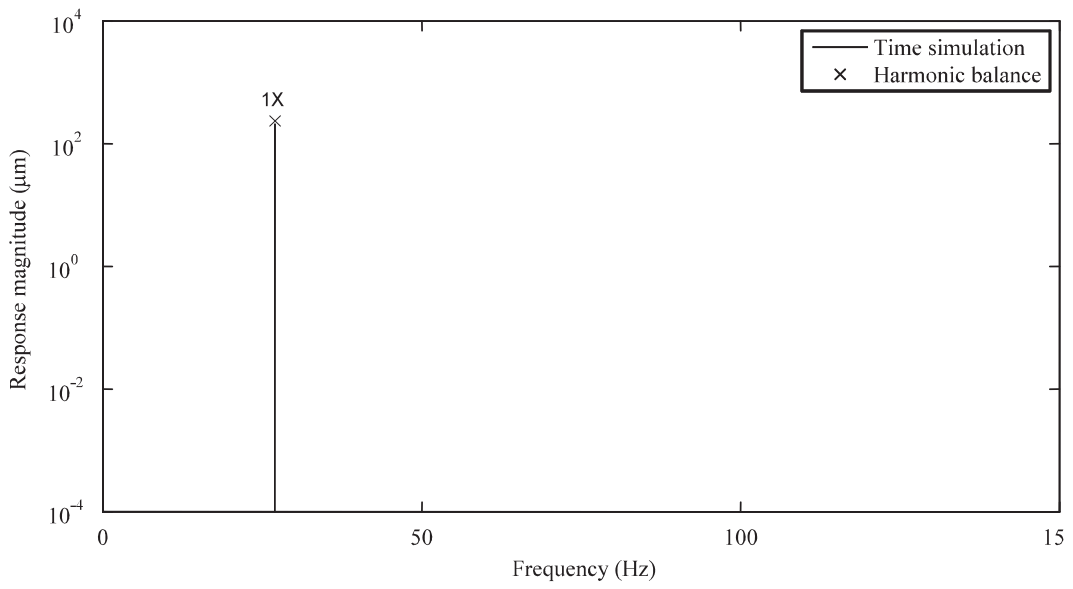

Fig. 7. The response of the uncracked rotor with no excitation.

only occurs at the rotor spin frequency (1X) and the AMB forcing frequency $(18 \mathrm{~Hz})$. A very good agreement between the time simulation and the harmonic balance results can be observed.

Suppose a crack is now added to the shaft. Two cases of the cracked shaft will be considered, modelled by assuming that the stiffnesses for the cracked element in two directions (perpendicular and parallel to the crack) are reduced by $25 \%$ and $12.5 \%$ (for the first case) and by $40 \%$ and $20 \%$ (for the second case). The crack opening and closing is assumed to be weight dominated, and hence the equations of motion are linear and parametrically excited. Figs. 9 and 10 show the responses for zero force from the AMB, for the $25 \%$ and $40 \%$ cracked shafts, respectively. The responses are at the rotor spin speed (1X) and its harmonics $(2 \mathrm{X}, 3 \mathrm{X}, 4 \mathrm{X}, 5 \mathrm{X})$, only. A very good agreement between the simulated and the harmonic balance results can be noticed. Comparing Figs. 9 and 10 one can see, that the deeper crack manifests itself through higher values of harmonics amplitudes.

Suppose the $25 \%$ cracked rotor is now excited by the AMB with a frequency of $\Omega_{2}=18 \mathrm{~Hz}$. According to Eqs. (11) and (22), for $s=1,0,1$ and $r=6,5, \ldots, 0, \ldots, 5,6$ we will obtain several values of combinational frequencies $\omega_{c}$ located at $9,18,27,36$, $45,54,63,72,81,90,9908,126$, and $144 \mathrm{~Hz}$. Fig. 11 presents the response of the $25 \%$ cracked shaft obtained by the time simulations and the harmonic balance method. The dominant frequency of the response spectrum is the spin speed of $27 \mathrm{~Hz}(1 \mathrm{X})$ and its $2 \mathrm{X}, 3 \mathrm{X}$, and $4 \mathrm{X}$ multiples with much smaller amplitudes. Moreover, the combinational frequency peaks (shown in italics) appear at the locations calculated above. It can be noticed that the frequencies and the amplitudes agree very well.

Although the frequency lines in Figs. 7-11 are very distinct, it should be emphasised that this is a very idealised case. A number of issues that arise in practice will now be discussed. The measurements should be taken at the steady state, and in the simulations the dampers were added to increase the damping in the first mode. In practice for lightly damped machines sufficient time must be allowed for the steady state to be reached. For the analysis, the computational time required for the harmonic balance is not affected by the damping, whereas for the time simulation the increased computational effort will be significant. 


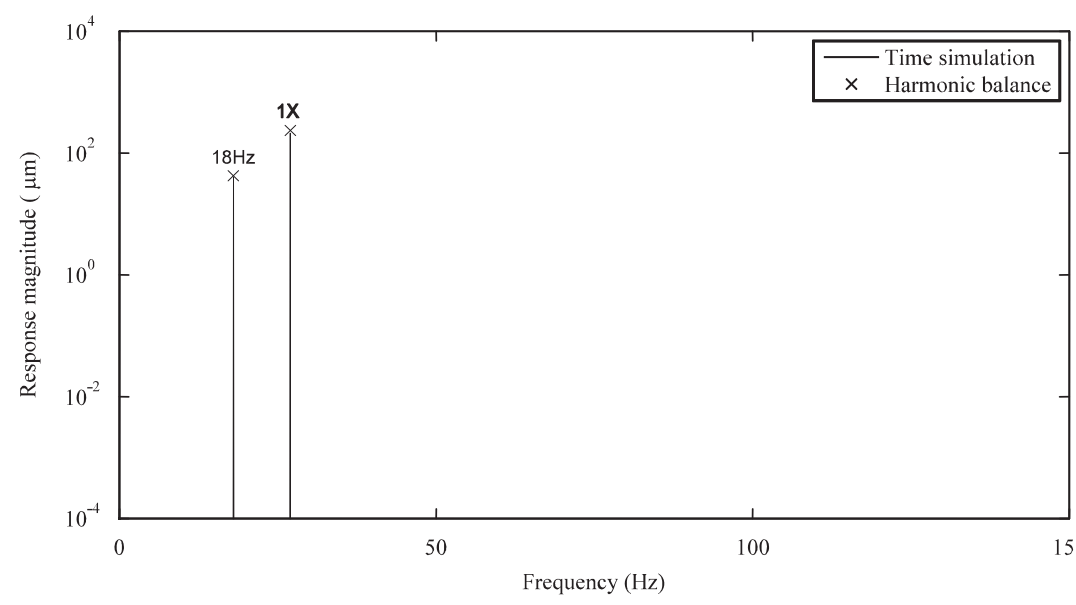

Fig. 8. The response of the uncracked rotor excited by the AMB $\left(\Omega_{2}=18 \mathrm{~Hz}\right)$.

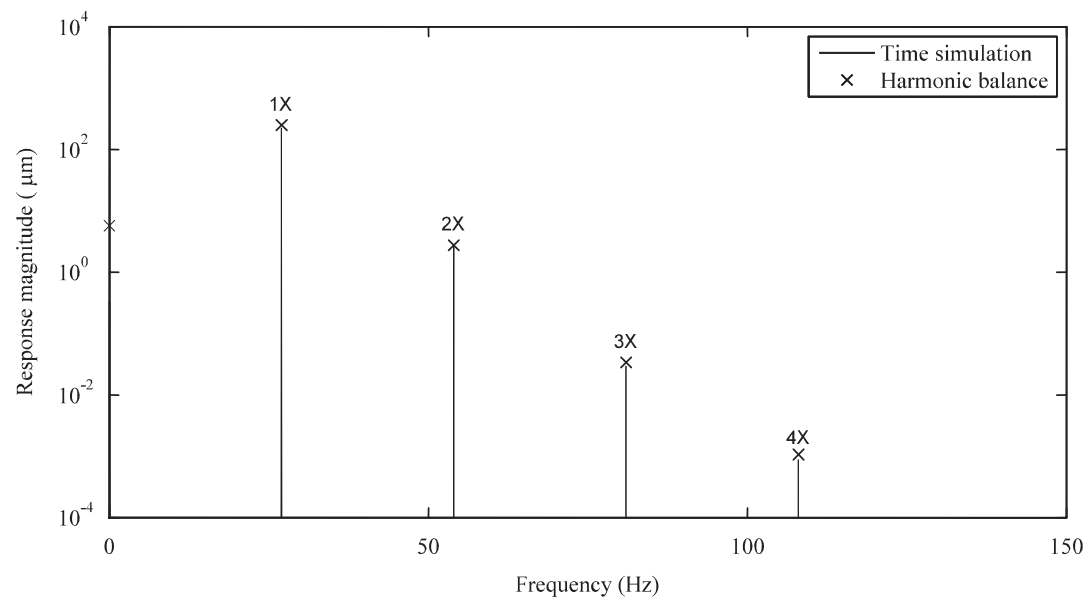

Fig. 9. The response of the $25 \%$ cracked rotor with no AMB excitation.

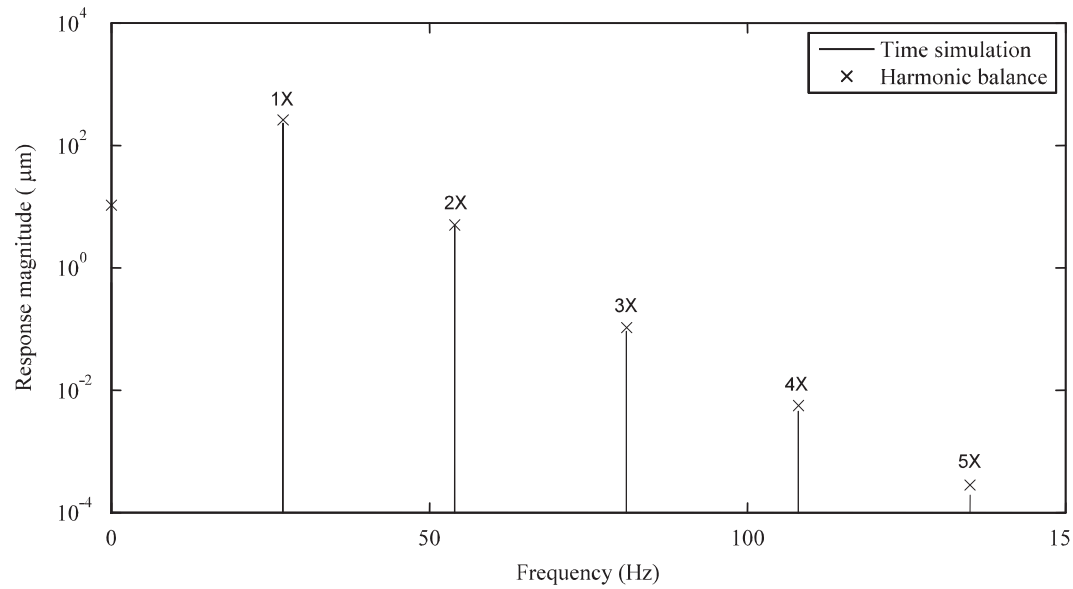

Fig. 10. The response of the $40 \%$ cracked rotor with no AMB excitation.

Consider the accuracy of the estimated critical frequency. Suppose the first critical frequency, which equals $\omega_{1}=36 \mathrm{~Hz}$, is estimated as $\tilde{\omega}_{1}=34 \mathrm{~Hz}$, giving the AMB frequency of $\Omega_{2}=20 \mathrm{~Hz}$, for $n=2$ in Eq. (7). Based on Eqs. (11), (22) the response $q_{d y}$ will include the combinational frequencies $\omega_{c}$ located at 7, 20, 27, 34, 47, 54, 61, 74, 81, 88, 101, and $108 \mathrm{~Hz}$. The effect of 


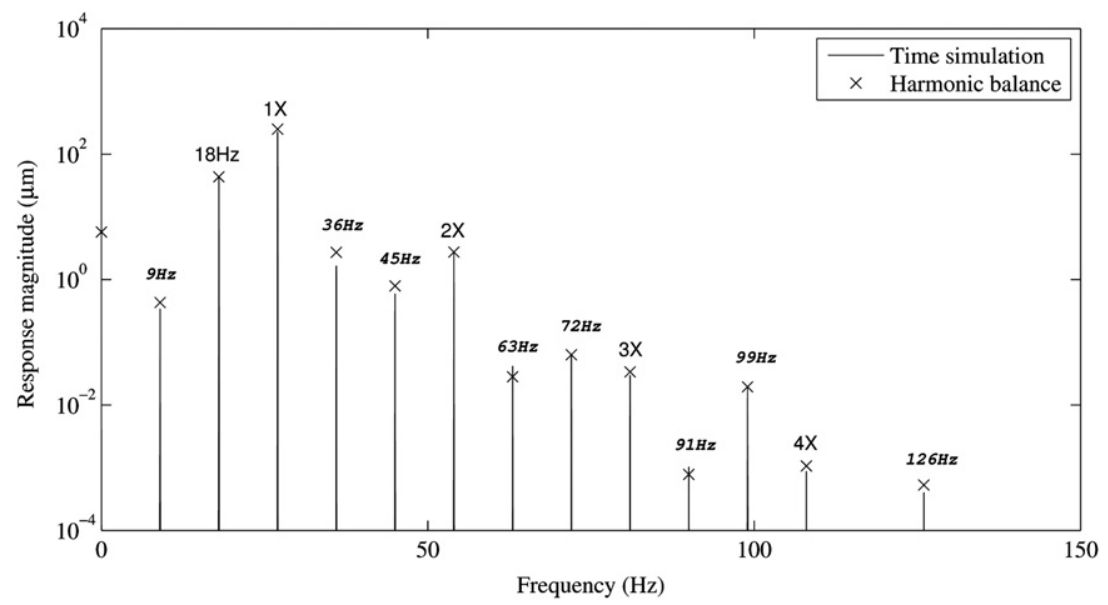

Fig. 11. The response of the $25 \%$ cracked rotor excited by the AMB $\left(\Omega_{2}=18 \mathrm{~Hz}\right)$.

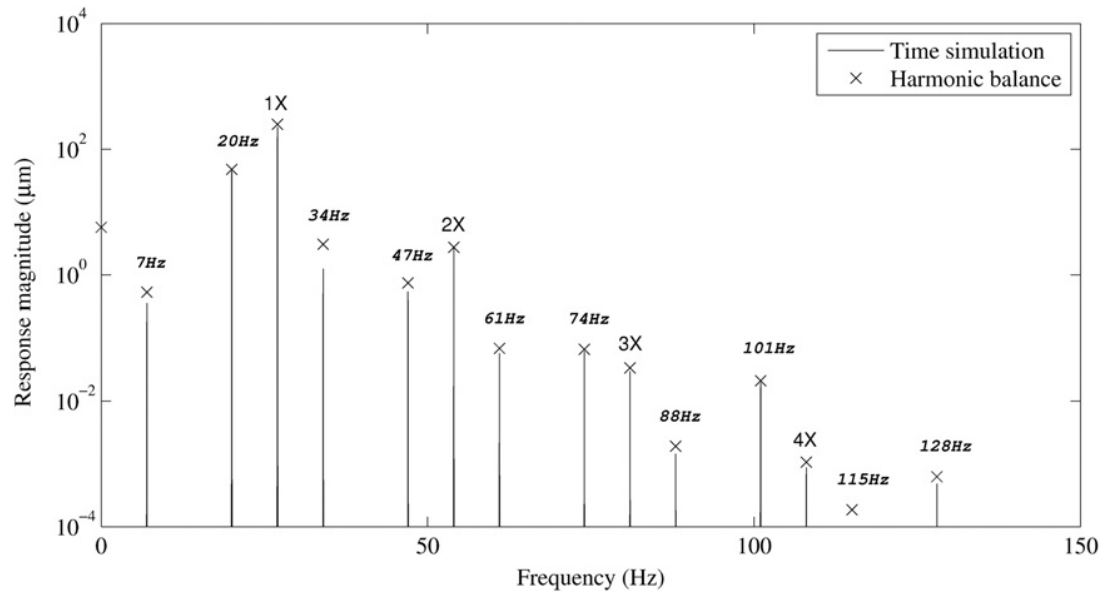

Fig. 12. The response of the $25 \%$ cracked rotor excited by the AMB $\left(\Omega_{2}=20 \mathrm{~Hz}\right)$.

estimating the natural frequency as $\tilde{\omega}_{1}=34 \mathrm{~Hz}$ is shown in Fig. 12. Apart from the rotor spin speed frequency and its multiplies, combinational frequencies occur at the values given above. The results obtained from the time simulations and the harmonic balance agree very well. Then suppose the first critical frequency is estimated as $\tilde{\omega}_{1}=38 \mathrm{~Hz}$, what for $n=2$ gives the AMB frequency of $\Omega_{2}=16 \mathrm{~Hz}$ (Eq. (7)). This time, according to Eqs. (11), (22), combinational frequencies will be located at 11, $16,27,38,43,54,65,70,81,92,97$ and $108 \mathrm{~Hz}$. Fig. 13 shows the response of the system for this case. Again, the results for the harmonic balance and the simulations agree very well and highlight that the natural frequencies do not have to be estimated very accurately.

Leakage is another problem in the calculation of the frequency domain response from the time data. Fig. 14 shows the effect when 271 cycles of the rotor spin frequency are used for the measurement. This means that the time period does not contain an integer number of cycles of the AMB forcing, and Fig. 14 clearly shows leakage effects around the combinational frequencies (compare to Fig. 11). It is possible to overcome the leakage effects since we know very accurately the rotor spin speed (assuming it is constant) and the AMB frequency; this allows us to choose the sample period, or alternatively we could use an estimator for the magnitude of the two frequency terms.

The harmonic balance approach shows that the amplitudes of the discreet combinational frequencies change while changing the excitation frequency. Fig. 15 shows the influence of the varying excitation frequency $\Omega_{2}$ on the response $\mathbf{q}_{\mathrm{dy}}$ for its different components $\mathbf{q}_{r s}$ (i.e. for different values of $r$ and s, Eqs. (11) and (22)). The different lines represent the amplitudes of different harmonics $\mathbf{q}_{r s}$. For $s=0$, i.e. when there is no AMB excitation (dot-dashed lines), the response and its harmonics do not depend on the external excitation frequency. For $s=1$ or 1, i.e. with the AMB exciting the rotor, the peaks of components $\mathbf{q}_{r s}$ can be observed at different frequencies of the AMB. The relatively high damping means that the peaks are quite wide. If the machine damping was low then they would be sharper. The peaks are located at the following excitation frequencies: 9 , $18,36,45,63,72 \mathrm{~Hz}$. All these frequencies fully satisfy condition (17) and apart from $36 \mathrm{~Hz}$ also condition (7). The highest peaks are near the excitation frequency of $36 \mathrm{~Hz}$, i.e. near the first critical speed $\omega_{1}$. This means that the amplitude of the rotor 


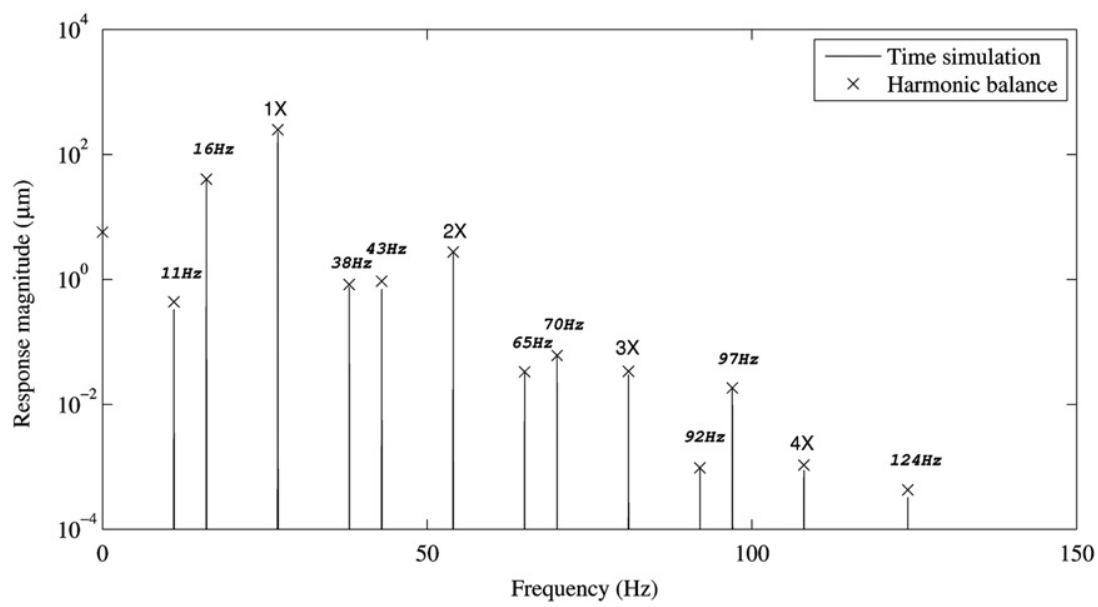

Fig. 13. The response of the $25 \%$ cracked rotor excited by the AMB $\left(\Omega_{2}=16 \mathrm{~Hz}\right)$.

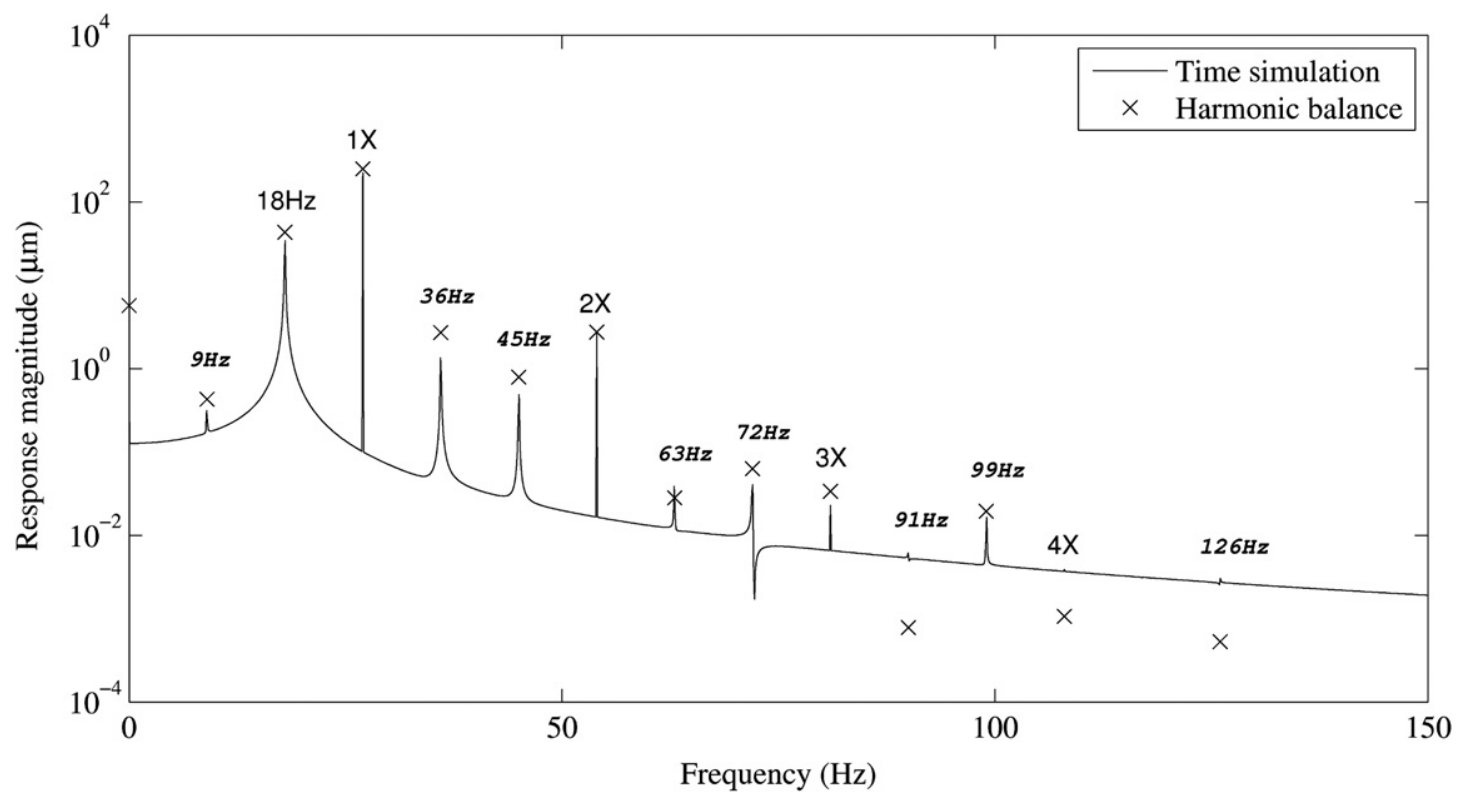

Fig. 14. The response of the $25 \%$ cracked rotor with leakage effects excited by the AMB $\left(\Omega_{2}=18 \mathrm{~Hz}\right)$.

response $\mathbf{q}_{\mathrm{dy}}$ will be maximum for this frequency too. The frequency spectrum of this response is presented in Fig. 16. Indeed, Fig. 16 compared with Figs. 11-13 shows higher amplitudes at the combinational frequencies. This is not a surprise, as $\Omega_{2}=36 \mathrm{~Hz}$ satisfies condition (17) (for $r=0$ and $s=1$ ). It would also satisfy condition ( 7 ) if parameter $n$ could be of value 0 . This shows that the multiple scale analysis can be seen as the subset of the harmonic balance method, as the results obtained from the multiple scales are consistent for some frequencies with the harmonic balance. However, for the experimental tests conducted so far, only excitation frequencies satisfying condition (7) were used. The results of these investigations are presented below.

Fig. 17 shows the response of the $40 \%$ cracked shaft obtained by the time simulations and experimental tests. The response frequencies from the simulation closely agree with those from the experimental data. Specifically, the running speed (1X) and its multiples (2X, 3X, 4X, 5X), AMB force excitation $(18 \mathrm{~Hz})$, critical frequency $(36 \mathrm{~Hz})$, and the combinational frequencies match well. The experimental data however, show amplitudes that are much lower and not so clearly defined compared to the simulation. The discrepancy in amplitudes has a number of likely causes, such as modelling errors including damping, small errors in the identification of the combination frequencies, poor estimation of the machine unbalance, errors in the force estimation from the AMB actuator, leakage effects in the experimental data, and difficulties in estimating the actual crack compliance. Note that the stiffness reduction due to the crack is estimated in a very simple way in our simulated example. Fig. 17 also shows that the measurements are contaminated with noise, although the amplitude is relatively small for the laboratory test rig. The effect of measurement noise will be larger for industrial plant, and also for machines where the 


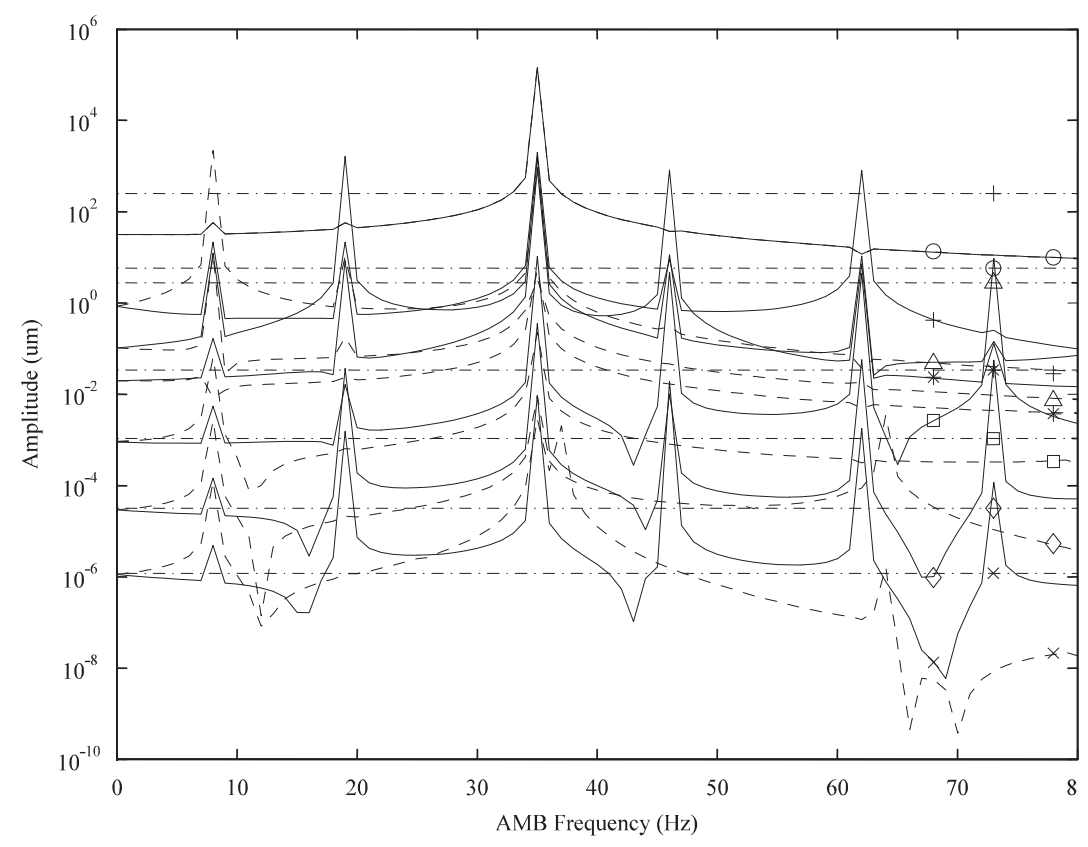

Fig. 15. The response of the $25 \%$ cracked rotor at different AMB frequencies. Line types: $s=1$ (solid), 0 (dot-dashed), or 1 (dashed), and $r=0$ (circle), 1 (cross), 2 (triangle), 3 (star), 4 (square), 5 (diamond), 6 (ex).

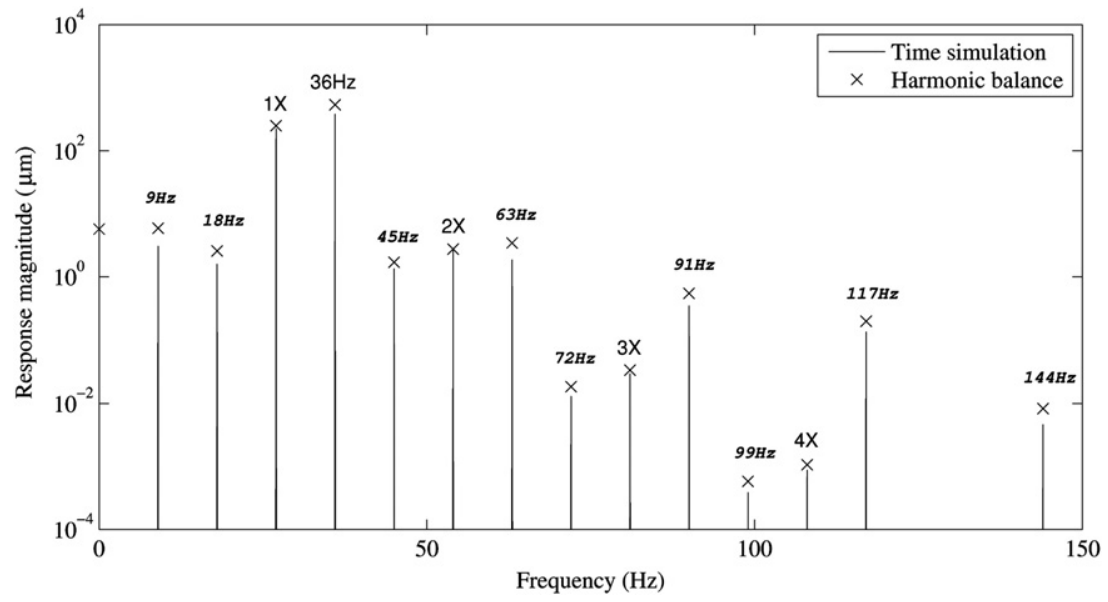

Fig. 16. The response of the $25 \%$ cracked rotor excited by the AMB $\left(\Omega_{2}=36 \mathrm{~Hz}\right)$.

response amplitude is small. Essentially there will be a noise floor in the measurements and only those combination frequencies whose response amplitude is above this noise floor will be detected, and this will have implications for real condition monitoring systems.

Thus far the AMB frequency has been calculated using $n=2$ and an estimate of the first critical frequency in Eq. (7). Suppose that $n=3$ with an estimated first critical frequency of $\omega_{1}=36 \mathrm{~Hz}$, giving an AMB frequency of $45 \mathrm{~Hz}$. Fig. 18 shows the spectrum in this case, which is very similar to Fig. 17 with the same combinational frequencies, although different response amplitudes. Fig. 19 shows the effect of using the first critical frequency $\left(\omega_{1}=36 \mathrm{~Hz}\right)$ and $n=4$ in Eq. (7), giving an AMB frequency of $72 \mathrm{~Hz}$. Clearly combinational frequencies are excited, and the character of the spectrum is similar.

Fig. 20 presents four responses of the uncracked rotor at different AMB frequencies obtained experimentally. The plots are shown in a vertical array so they can be visually compared. The first plot in Fig. 20 shows the responses without AMB excitation. No combinational frequencies could be observed there. The trials with AMB excitation are plotted in the last three plots and have the features unique to each of them. AMB signal excitation appears as the peak in the response spectrum at the corresponding frequency. The second plot of the figure has an AMB excitation peak at $18 \mathrm{~Hz}$, the third plot at $45 \mathrm{~Hz}$, and the fourth plot at $72 \mathrm{~Hz}$. The plots with the AMB excitation show traces of combinational frequencies (labeled in italics), but their amplitudes are negligible. 


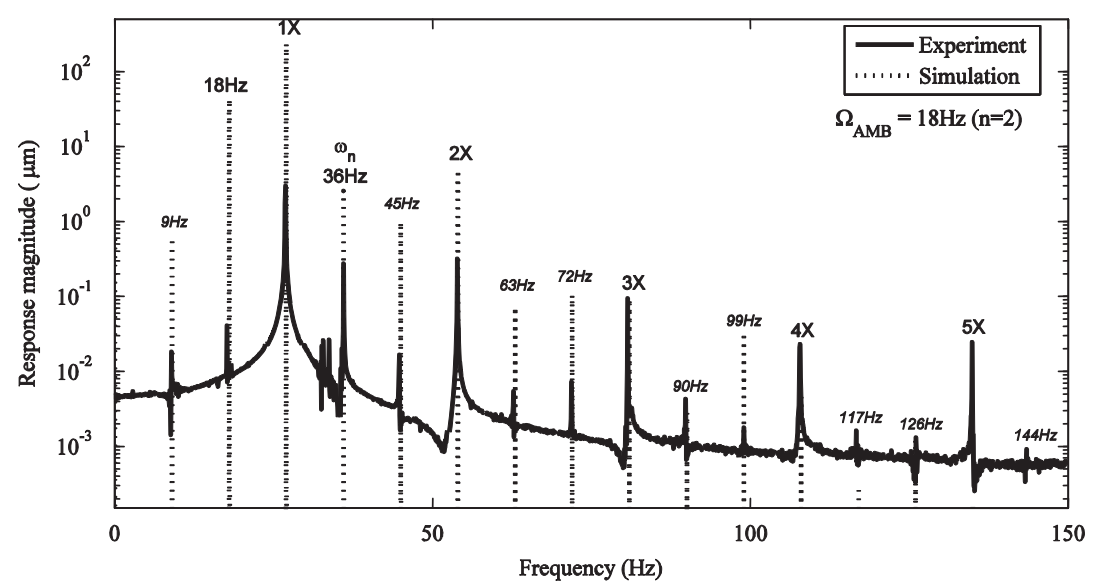

Fig. 17. The response of the $40 \%$ cracked rotor excited by the AMB $\left(\Omega_{2}=18 \mathrm{~Hz}\right)$.

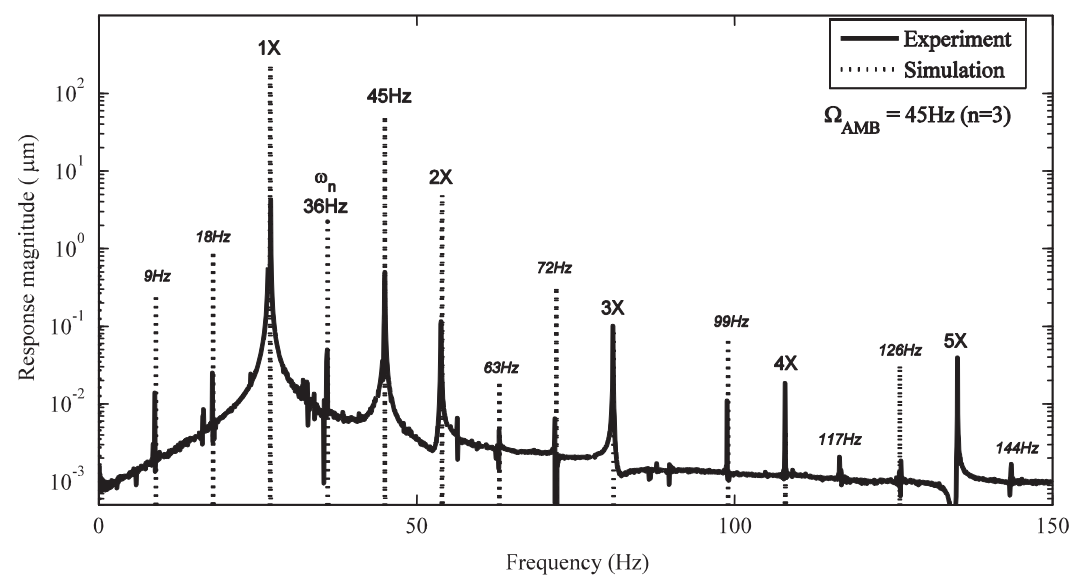

Fig. 18. The response of the $40 \%$ cracked rotor excited by the AMB $\left(\Omega_{2}=45 \mathrm{~Hz}\right)$.

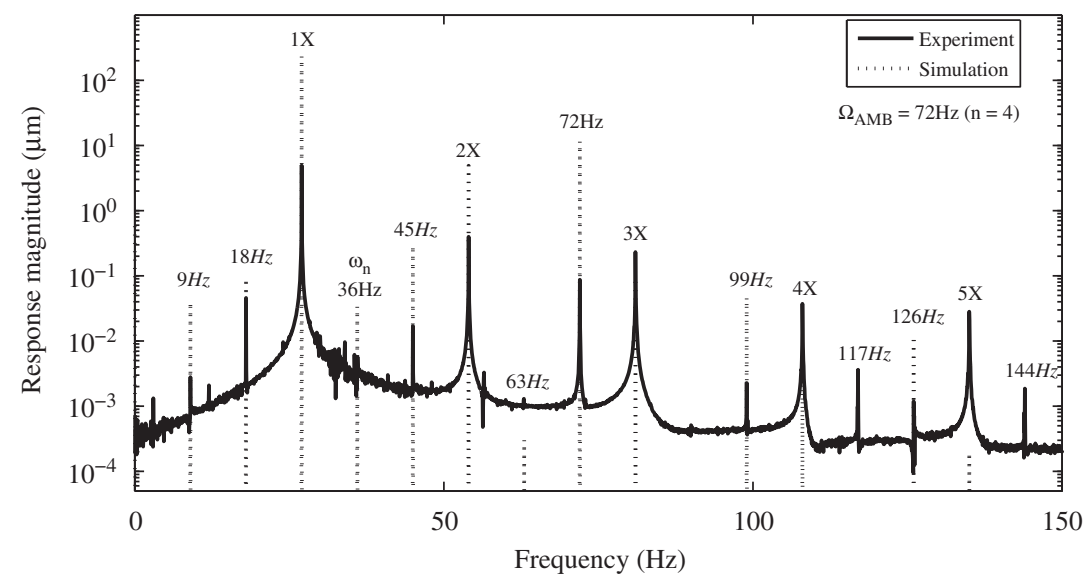

Fig. 19. The response of the $40 \%$ cracked rotor excited by the AMB $\left(\Omega_{2}=72 \mathrm{~Hz}\right)$.

Fig. 21 presents four experimental responses at different AMB frequencies, similar to those presented in Fig. 20, but for the $25 \%$ cracked rotor. This time, the last three plots with AMB excitation show a significant difference in the combinational frequency content and amplitudes in comparison with the healthy shaft case. It is evident that combinational frequency peaks (shown in italics) are more common as well as appear larger in amplitude, due to the presence of the $25 \%$ crack. Also, the magnitudes of the responses at the induced combinational frequencies are larger for higher excitation frequencies. 

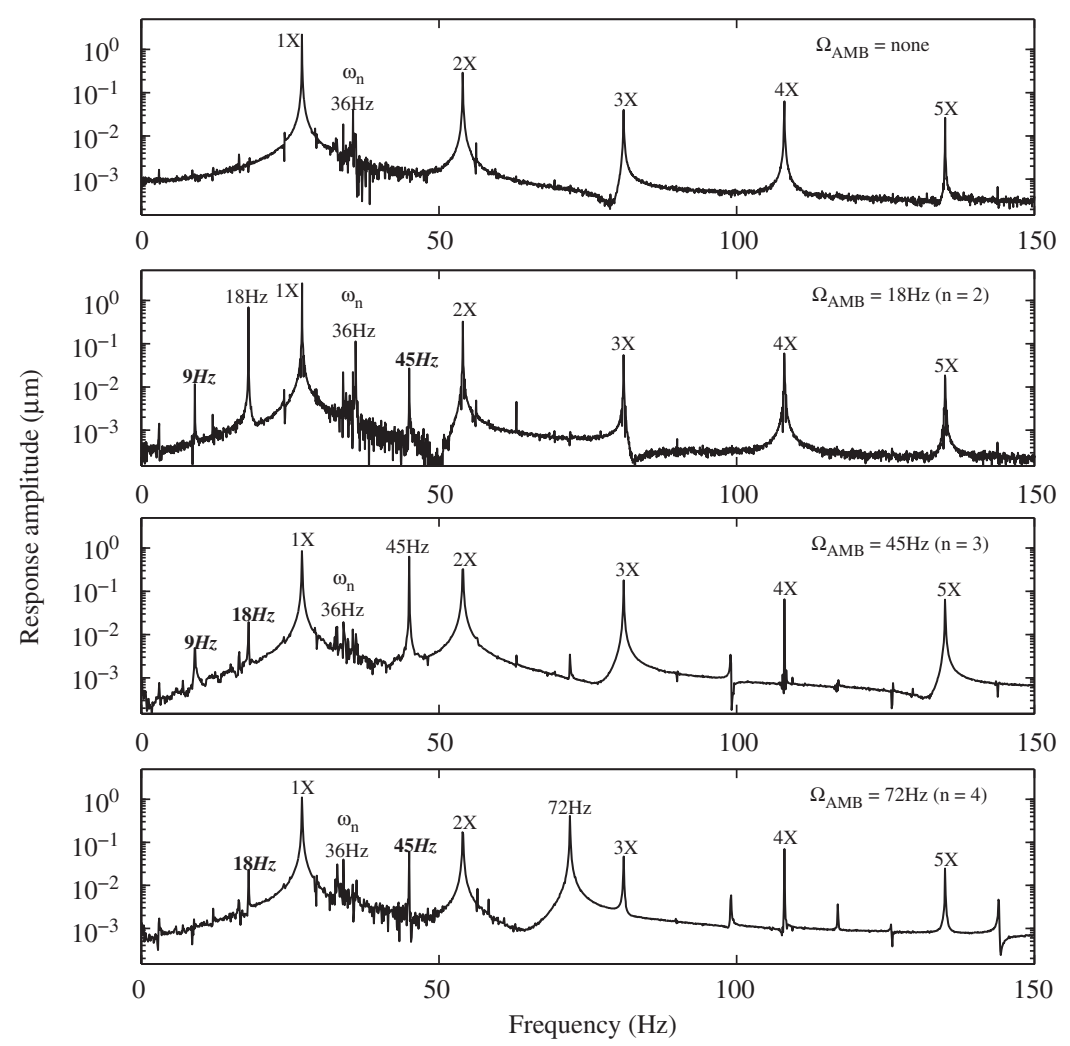

Fig. 20. The responses of the uncracked rotor at different AMB frequencies.

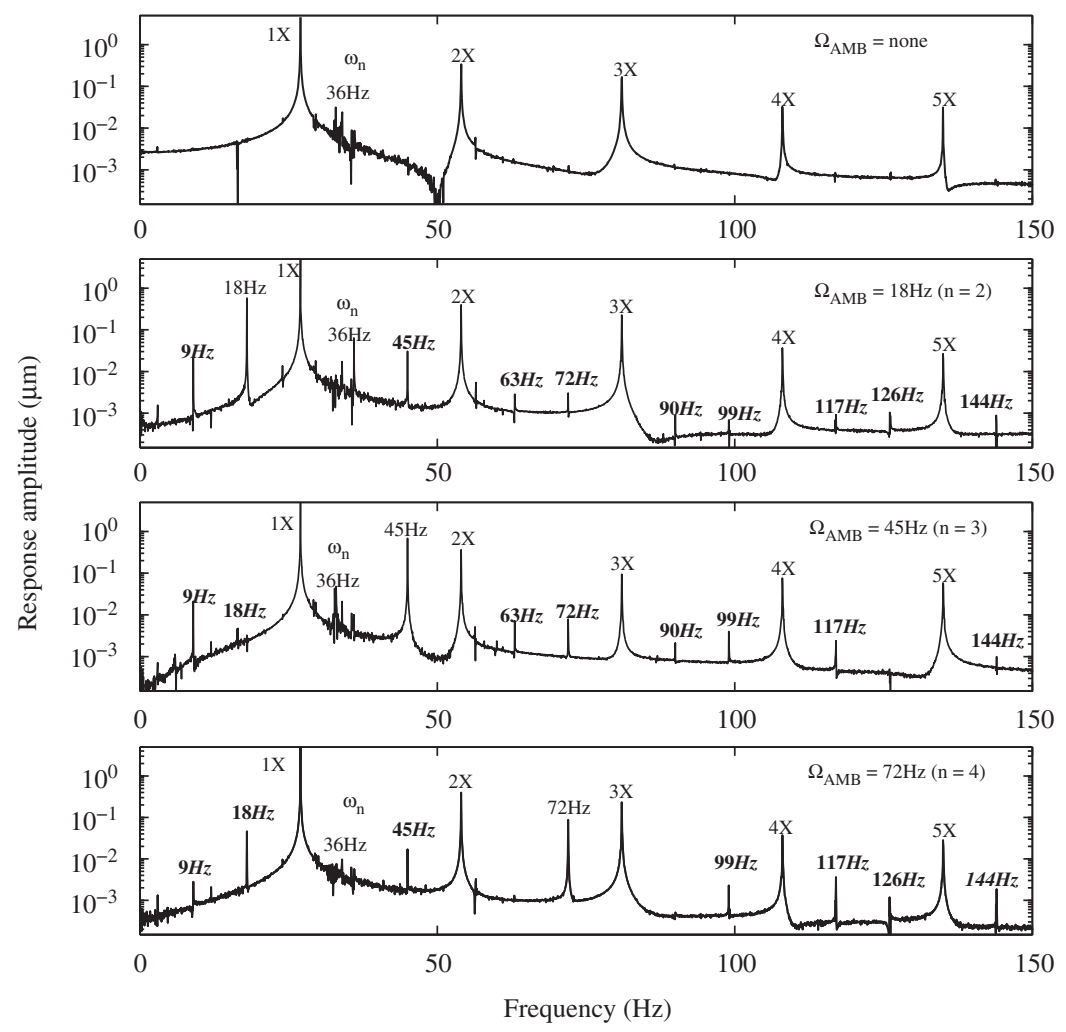

Fig. 21. The response of the $25 \%$ cracked rotor excited by the AMB with various frequencies. 


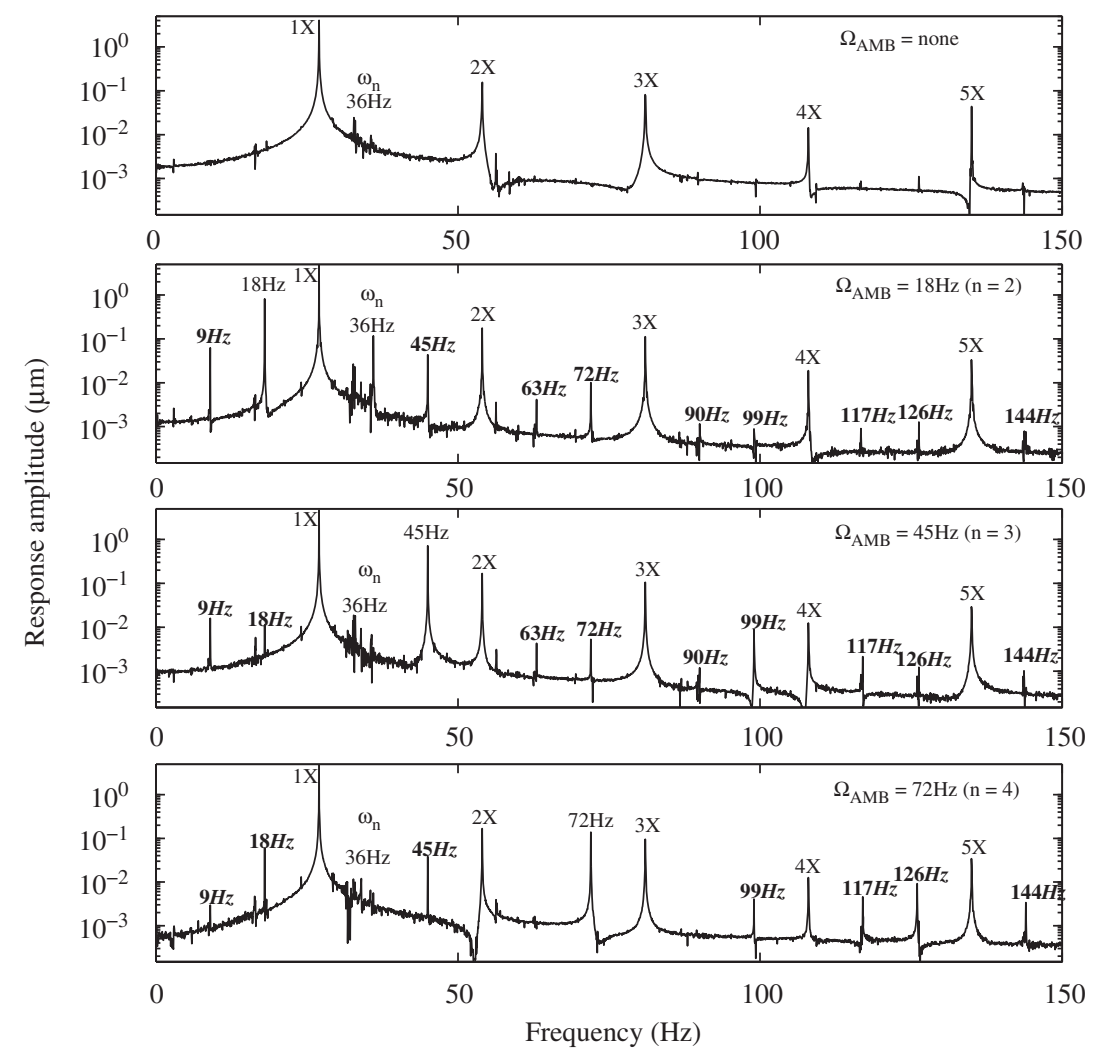

Fig. 22. The response of the $40 \%$ cracked rotor excited by the AMB with various frequencies.

Fig. 22 presents the similar results as those in Figs. 20 and 21, but for the $40 \%$ cracked rotor. The responses show similar combinational frequency content but with an increasing trend in amplitude when compared to the $25 \%$ cracked shaft. The three plots with AMB excitation essentially contain all the combinational frequencies. Also, the magnitudes of the responses at the induced combinational frequencies are larger for higher excitation frequencies.

It is clear from these examples that the cracked rotor response at the combinational frequencies can be used to detect that the system is parametrically excited. However, estimation of the state of the rotor and obtaining more details of its condition requires the analysis of the relative amplitudes of the response at these different frequencies. The response calculation based on the harmonic balance method is sufficiently fast to allow such analysis. However, difficulties exist because of signal to noise ratio issues (although averaging can help, provided that the rotor spin speed is held constant) and the sensitivity of the response amplitudes to different damage mechanisms.

\section{Conclusions}

A variety of approaches have been proposed to try to make use of the changes in the dynamics of a rotor to identify and possibly locate crack (and other faults) in a rotor at an early stage in their development. The simulations shown suggest that the use of an auxiliary active magnetic bearing (AMB) to help identify crack in the rotor has some merit, but further work is needed to produce a robust condition monitoring technique. Applying a sinusoidal force from the AMB produces combinational frequencies based on the AMB frequency, and the rotational speed, that could be used to detect cracks in the rotor. However, a robust method is needed to determine the presence, location and severity of a crack from the combinational frequencies in the response. The harmonic balance method proposed in this paper provides a fast and convenient method to predict the responses, and could be used with inverse methods for damage detection. Furthermore, the effect of adding an extra force to the system might encourage a faster crack growth, which is obviously a disadvantage.

\section{Acknowledgment}

This research has been funded by NASA's Research Opportunities in Aeronautics, Grant no. NNX08AC31A. 


\section{References}

[1] P. Pennacchi, N. Bachschmid, A. Vania, A model-based identification method of transverse cracks in rotating shafts suitable for industrial machines, Mechanical Systems and Signal Processing 20 (8) (2006) 2112-2147.

[2] N. Bachschmid, P. Pennacchi, E. Tanzi, A. Vania, Identification of transverse crack position and depth in rotor systems, Meccanica 35 (2000) $563-582$.

[3] R. Markert, R. Platz, M. Seidler, Model based fault identification in rotor systems by least squares fitting, International Journal of Rotating Machinery 7 (5) (2001) 311-321.

[4] Z. Kulesza, J.T. Sawicki, Auxiliary state variables for rotor crack detection, Journal of Vibration and Control 2010, doi:10.1177/1077546309360050.

[5] A.D. Dimarogonas, Vibration of cracked structures: a state of the art review, Engineering Fracture Mechanics 55 (1996) $831-857$.

[6] W. Ostachowicz, M. Krawczuk, On modeling of structural stiffness loss due to damage, DAMAS 2001: 4th International Conference on Damage Assessment of Structures, Cardiff, 2001, pp. 185-199.

[7] M.I. Friswell, J.E.T. Penny, Crack modelling for structural health monitoring, Structural Health Monitoring: An International Journal 1 (2) (2002) 139-148.

[8] C.A. Papadopoulos, A.D. Dimarogonas, Coupled longitudinal and bending vibrations of a rotating shaft with an open crack, Journal of Sound and Vibration 117 (1) (1987) 81-93.

[9] O.S. Jun, H.J. Eun, Y.Y. Earmme, C.-W. Lee, Modelling and vibration analysis of a simple rotor with a breathing crack, Journal of Sound and Vibration 155 (2) (1992) $273-290$.

[10] I.W. Mayes, W.G.R. Davies, The vibration behaviour of a rotating shaft system containing a transverse crack, International Conference on Vibrations in Rotating Machinery, Paper C168/76, IMechE, 1976, pp. 53-64.

[11] H. Keiner, M.S. Gadala, Comparison of different modelling techniques to simulate the vibration of a cracked rotor, Journal of Sound and Vibration 254 (5) (2002) 1012-1024.

[12] N. Bachschmid, P. Pennacchi (Eds.), Crack effects in rotordynamics, Special Issue of Mechanical Systems and Signal Processing 22 (4) (2008) $761-904$.

[13] T. Iwatsubo, S. Arii, A. Oks, Detection of a transverse crack in a rotor shaft by adding external force, Proceedings of the International Conference on Vibrations in Rotating Machinery, Paper no. C432/093, IMechE, 1992, pp. 275-282.

[14] Y. Ishida, T. Inoue, Detection of a rotor crack by a periodic excitation, Proceedings of the ISCORMA, 2001, pp. 1004-1011.

[15] G. Mani, D.D. Quinn, M.E.F. Kasarda, D.J. Inman, R.G. Kirk, Health monitoring of rotating machinery through external forcing, in: J.T. Sawicki, A. Muszynska (Eds.), Proceedings of the Third International Symposium on Stability Control of Rotating Machinery, ISCORMA-3, 2005.

[16] G. Mani, D.D. Quinn, M. Kasarda, Active health monitoring in a rotating cracked shaft using active magnetic bearings as force actuators, Journal of Sound and Vibration 294 (2005) 454-465.

[17] D.D. Quinn, G. Mani, M.E.F. Kasarda, T.J. Bash, D.J. Inman, R.G. Kirk, Damage detection of a rotating cracked shaft using an active magnetic bearing as a force actuator-analysis and experimental verification, IEEE/ASME Transactions on Mechatronics 10 (6) (2005) 640-647.

[18] Y. Ishida, T. Inoue, Detection of a rotor crack using a harmonic excitation and nonlinear vibration analysis, ASME Journal of Vibration and Acoustics 128 (2006) 741-749.

[19] J.T. Sawicki, M.I. Friswell, A.H. Pesch, A. Wroblewski, Condition monitoring of rotor using active magnetic actuator, Paper GT2008-5116, Turbo ASME Turbo Expo Conference, Berlin, Germany, 9-13 June 2008.

[20] J.T. Sawicki, A.K. Sen, G. Litak, Multiresolution wavelet analysis of the dynamics of a cracked rotor, International Journal of Rotating Machinery, (2009) doi: $10.1155 / 2009 / 265198$.

[21] H. Ecker, A. Tondl, Stabilization of a rigid rotor by a time-varying stiffness of the bearing mounts, International Conference on Vibrations in Rotating Machinery, Paper C623/014/2004, IMechE, 2004, pp. 45-54.

[22] R.A. Gasch, Survey of the dynamic behavior of a simple rotating shaft with a transverse crack, Journal of Sound and Vibration 160 (1993) 313-332.

[23] I.W. Mayes, W.G.R. Davies, A method of calculating the vibrational behaviour of coupled rotating shafts containing a transverse crack, IMechE International Conference on Vibrations in Rotating Machinery, Paper C254/80, 1980, pp. 17-27.

[24] I.W. Mayes, W.G.R. Davies, Analysis of the response of a multi-rotor-bearing system containing a transverse crack in a rotor, ASME Journal of Vibration, Acoustics, Stress, and Reliability in Design 106 (1984) 139-145.

[25] J.E.T. Penny, M.I. Friswell, Simplified modelling of rotor cracks, ISMA 27, Leuven, Belgium, 2002, pp. 607-615.

[26] J.E.T. Penny, M.I. Friswell, C. Zhou, Condition monitoring of rotating machinery using active magnetic bearings, ISMA 2006, Leuven, Belgium, 2006, pp. 3497-3506.

[27] J.E.T. Penny, M.I. Friswell, The dynamics of cracked rotors, IMAC XXV, Orlando, FL, paper 198, 2007.

[28] M.I. Friswell, J.E.T. Penny, The accuracy of jump frequencies in series solutions of the response of a Duffing oscillator, Journal of Sound and Vibration 169 (1994) 261-269.

[29] J.J. Thomsen, Vibrations and Stability: Order and Chaos, McGraw-Hill, Maidenhead, 1997. 